\title{
Pferde: Neu oder gebraucht?
}

\author{
Heinz Meyer
}

\begin{abstract}
Zusammenfassung:
Um der Richtlinie der Europäischen Union für den Verbrauchsgüterkauf zu entsprechen, traten in Deutschland am 1.1.2002 neue gesetzliche Bestimmungen über den Kauf von Sachen in Kraft. Mit der EU-Richtlinie wird vor allem bezweckt, Verbraucher beim Kauf von Gebrauchsgütern von Unternehmern zu schützen. Die Richtlinie sowie die ihr entsprechenden Gesetze betreffen unter anderem den Kauf von Pferden. Die früheren deutschen Sonderbestimmungen für den Viehkauf wurden ersatzlos aus dem Bürgerlichen Gesetzbuch gestrichen. Die neuen Gesetze enthalten eine weitgehende Haftungsverpflichtung für den gewerblichen Verkäufer, nämlich die Haftung auf die Daver von zwei Jahren bei neuen und von einem Jahr bei gebrauchten Gütern. Die gewerblichen Verkäufer von Pferden werden in Zukunft vermutlich häufiger als in der Vergangenheit versuchen, sich im Rahmen der Kaufuntersuchung bestimmte Eigenschaften der Pferde bescheinigen zu lassen und die Tierärzte derart als Garanten dieser Eigenschaften gegenüber dem Käufer in den Handelsprozess einzubeziehen. Das wird unter anderem zu dem Zweck geschehen, in dem Fall Schadenersatzansprüche an den Tierarzł stellen zu können, in dem der Verkäufer gegenüber dem Käufer für Mängel eines Pferdes zu haften hat und unvollständige oder unzutreffende Aussagen des Tierarztes über die Beschaffenheit und/oder die Verwendbarkeit des Pferdes die Entscheidung des Käufers mitbestimmten. Die Käufer, die ihr Pferd von einem professionellen Verkäufer erwerben, werden dementsprechend nach dem neven Recht in deutlich stärkerem Maße als nach dem alten geschütz†; sie bedürfen daher nicht in dem Maße wie zuvor der Prüfung der vom Verkäufer zugesicherten Eigenschaften in einer Kaufuntersuchung durch den Tierarzt.Angesichts des Zeitraums der unumgänglichen Haftung (des professionellen Verkäufers gegenüber dem Käufer) von zwei Jahren bei neuen "Sachen" und von einem Jahr bei gebrauchten gewinnt die Bestimmung von adulten Pferden, von Fohlen, von Ei- und Samenzellen sowie von deren Verbindung als "neu" einerseits oder als "gebraucht" andererseits für Verkäufer sowie für Käufer und indirekt auch für Tierärzte Relevanz. Sprachlich und sachlich ist die Übertragung der für die Beschreibung lebloser Verbrauchsgüter geeigneten Begriffe "neu" und "gebraucht" auf Lebewesen eigentlich nicht korrekt. In absehbarer Zeit werden die Gesetze wahrscheinlich aber nicht novelliert werden. Daher muss die Rechtspraxis gerichtliche Auseinandersetzungen mit Hilfe von grundsätzlichen Argumentationen und Einzelfallentscheidungen pragmatisch lösen. Zur Frage des Neu- oder des Gebrauchtseins der Lebewesen resultiert aus den vorliegenden Überlegungen: Pferde, Fohlen sowie Ei- und Samenzellen sind keine gebrauchsfertigen neu hergestellten Verbrauchsgüter im Sinne der EU-Richtlinie. Diese Erkenntnis zwingt dazu, Pferde, Fohlen sowie deren Zellen der einzigen im Gesetz vorgesehenen Alternative zuzuordnen, nämlich den "gebrauchten" Gütern. Pferde werden erst nach ihrer Ausbildung unter dem Sattel und/oder vor dem Wagen für ihren bestimmungsgemäßen Zweck gebrauchsfertig. Die generelle Zuordnung zu den gebrauchten Gütern ist insofern "sachlich" gerechtfertigt, als Lebewesen schon in ihrer Existenz als einzelne Zellen keine - den leblosen Verbrauchsgütern nach ihrer Herstellung und vor ihrem Gebrauch ähnliche - statischen Sachen darstellen, als sie sich vielmehr laufend verändern und diversen Einflüssen unterliegen, und zwar Einflüssen, die sich in der Nutzungskapazität der adulten Individuen auswirken und die insofern dem "Gebrauch" der Verbrauchsgüter zu parallelisieren sind, auch wenn es sich bei einem solchen "Gebrauch" nicht um den bestimmungsgemäßen, nämlich den unter dem Sattel oder vor dem Wagen, handelt. Ausnahmen bilden die für den Verzehr gezüchteten und die ausschließlich als Sozialpartner oder als lebende "Rasenmäher" eingesetzten Pferde. Diese gewinnen ihre Gebrauchsfertigkeit nämlich im Verlaufe ihres Wachstumsprozesses, das heißt ohne besondere Einwirkung des Menschen, speziell ohne die Einwirkung im Rahmen einer Ausbildung, die die Voraussetzung einer differenzierten Nutzung darstellt. Gleichwohl sind solche Pferde in ihren frühen Entwicklungsstufen ebenfalls Einflüssen und Einwirkungen ausgesetzt, die sich in den Lebewesen niederschlagen und einen potentiellen "Neu"-Zustand aufheben. Pferde sind also in keinem Fall "neu" im Sinne des Verbrauchsgüterkaufrechts.
\end{abstract}

Schlüsselwörter: Pferdekauf, Recht, Kaufuntersuchung, Haftung, neu, gebraucht

\section{Horses - new or used?}

On the 1.1.2002 the German legislation about buying consumer goods was changed. It was done for the purpose of correspondence with the directive of the European Union about buying consumer goods. The aim of the directive was to protect the consumers in buying goods from professional dealers. The EU-directive and its implementation in the German legislation among other things refers to the buying of horses. The former special German laws about buying animals became annulated. The new German legislation includes guarantee periods, during which the professional seller is liable to the buyer for the quality and the function of the consumer goods, these are two years for new goods and one year for used. In the future the professional sellers of horses will be hold more often than in the past responsible for defects of bought horses. And these sellers will try more often than in the past, to involve the veterinarians in the buying process with their purchase examinations and to sue them for damages in the case, in which the buyer makes the seller responsible for a wrong promise of special qualities of the horse, which he bought. On the other hand: Buying from professional dealers consumers now are more protected than in the past; that means, they don't need as much as before the certification of the capacities of a horse in a purchase examination. Regarding the liability of two years for new goods and the liability of one year for used goods there is an importance to determine a horse, a foal or a cell as "new" or as "used". Regarding the usual speech and the speciality of the different things the translation of the terms "new" and "used", which are suitable to describe lifeless consumer goods, to animals is not correct. The laws will probably not be modified in the near future. Therefore the jurisdiction has to find principal argumentations and special decisions to solve legal actions. The result of the here made discussion: Horses, foals and also the cells are not alike new made products ready for use, as which the directive of the European Union deals with. This evidence obliges to subsume adult horses, foals and their cells to used consumer goods, which are in the law the only alternative to the new products. Regarding the reality this subsumtion does make sense, because living cells and living individuals can not be considered as static objects; they are permanently modifying themselves and they are exposed to different influences. These influences are affecting the later proper use capacity of the horses in riding and coaching; in this way the early 
influences correspond to the use of consumer goods. Exceptions are the horses, which are breed for slaughtering, and the horses, which are used only as social companions or living lawn-movers. Those horses get ready for use while growing up, it means without human work as the schooling, which is conditional for a differentiated use of a horse. But those horses are in the same way as other horses exposed to influences in their early stages. These influences have consequences in the individuals and so they abandon the potential status to be new. This means: Horses are in no case new. They get ready for their proper use not before they are specially trained.

Keywords: horse buying, legislation, purchase examination, liability, new, used

Das für den Pferdekauf gültige deutsche Recht hat sich seit dem Anfang des Jahres 2002 in weiten Bereichen geändert. Die Modifizierung resultierte aus der Anpassung des deutschen Rechts an die Richtlinie 1999/44 (Verbrauchsgüterkauf) der Europäischen Union. Am 1. Januar 2002 trat das sogenannte "Schuldrechtmodernisierungsgesetz" in Deutschland in Kraft. Die Rede vom "völlig neuen Pferdekaufrecht" (Fellmer et al. 2002) vermittelt allerdings einen irrtümlichen Eindruck. Es gibt nämlich seit dem Anfang dieses Jahres in Deutschland kein spezielles "Pferdekaufrecht" mehr; zudem betreffen die durch die Anpassung an die Richtlinie der Europäischen Union bedingten gesetzlichen Veränderungen nicht alle Verkäufe, sondern vor allem die des Unternehmers an den (End)Verbraucher. Modifiziert wurden insbesondere die Bestimmung von Mängeln, die Beweislast beim Vorhandensein von Mängeln sowie die Haftung für Mängel. Mit diesen Veränderungen verschiebt sich im Rahmen des Pferdehandels unter anderem in verschiedenen Punkten die Bedeutung der tierärztlichen Kaufuntersuchung und die vom Tierarz† für die Aussagen seines Gutachtens zu übernehmenden Haftung. Eine grundlegend veränderte Rolle hat die Kaufuntersuchung durch die Neufassung des Kaufrechts laut Plewa (2002,284 ss.) allerdings nicht erhalten.

Die Bedeutung der Kaufuntersuchung kann sich vor allem in den Fällen modifizieren, in denen ein professioneller Verkäufer sich bei der Zusicherung der Beschaffenheit des offerierten Pferdes auf die Aussagen des (von ihm beauftragten) Tierarztes stützt, in denen ein Mangel des Pferdes offenbar wird und der Verkäufer für diesen haften muss. Relevant ist für solche Fälle: Die Daver der Haftung des Verkäufers wurde in den von den veränderten Gesetzen betroffenen Käufen erheblich ausgeweitet, und zwar auf ein beziehungsweise zwei Jahre ab dem Zeitpunkt der Übernahme des gekauften Pferdes. Für den Tierarzt hat sich demnach das Risiko erhöht, (im Fall eines berechtigten Anspruchs des Käufers) für unvollständige oder unzutreffende gutachterliche Aussagen, die aus Kunstfehlern oder mangelnder Sorgfalt resultieren, gegenüber dem Auftraggeber, nämlich dem Verkäufer des Pferdes, Schadenersatz leisten zu müssen. Insofern kann sich die Dauer der Haftung des Verkäufers, nämlich zwei Jahre bei einem Pferd, das als "neu" klassifiziert wird, und ein Jahr, wenn das zur Diskussion stehende Pferd ein "gebrauchtes" Gut darstellt, in den möglichen Folgen einer Kaufuntersuchung niederschlagen. Die Frage, ob beziehungsweise wann ein Pferd als "neu" oder als "gebraucht" zu bestimmen ist, stellt sich damit nicht nur für den "Konsumenten" und den professionellen Verkäufer, sondern indirekt, nämlich aufgrund möglicher Schadenersatzansprüche durch den Auftraggeber, ebenfalls für den Tierarzt. Auf die Kaufuntersuchung selbst und auf die grundsätzliche Haftung des Tierarztes gegenüber seinem Auftraggeber wirkt sich die Qualifizierung eines Pferdes als "neu" oder als "gebraucht" aber nicht aus.

\section{Verbrauchsgüter und Unternehmer}

Die Veränderungen im deutschen Recht entsprechen der EURichtlinie 1999/44 zum Kauf von Verbrauchsgütern. Mit dieser Richtlinie sollen Käufer von Verbrauchsgütern geschütz† werden; sie sollen vor allem vor Verkäufern geschütz† werden, die mit aggressiven und trickreichen Methoden mangelhafte Ware veräußern, nämlich Ware, die aufgrund ihrer Mängel nicht den Zweck erfüllt, für den die Käufer sie erwarben. (Hoeren 2002,561) Die Richtlinie betrifft also in erster Linie Verbrauchsgüter und den Handel zwischen dem "Unternehmer" - hier in der Regel der professionell mit Pferden beschäftigte Händler, Ausbilder oder Züchter - und (s)einem privaten Kunden, nämlich dem "Verbraucher". Sie betrifft eigentlich nicht das Kaufen von Sachen im allgemeinen. Dem Anliegen der EU-Richtlinie entspricht die Neufassung des Bürgerlichen Gesetzbuches(§ 474 ss.) insofern, als dort vom "Verbrauchsgüterkauf" die Rede ist und dieser als der Kauf einer "beweglichen Sache", nämlich eines Verbrauchsgutes, durch einen "Verbraucher" von einem (professionell agierenden) "Unternehmer" bestimmt wird. Bei einem derartigen Kauf beziehungsweise Verkauf einer Ware wird der Käufer insofern gegen (den Gebrauch mindernde oder verhindernde) Mängel der Ware geschützt, als der Verkäufer zwei beziehungsweise ein Jahr für Mängel des erworbenen Gutes zu haften hat. Die Haftung für Mängel eines Verbrauchs- respektive Gebrauchsgutes ist als Haftung für die (vereinbarte oder die allgemein erwartbare) Gebrauchsfähigkeit dieses Gutes zu verstehen. Den im Fall des (eng definierten) "Verbrauchsgüterkaufs" gesetzlich garantierten Schutz gewinnt nicht der Käufer, der ein Gebrauchsgut bei einem (nicht als "Unternehmer" tätigen) Privatmann erwirbt. Ferner gewinnt ihn nicht der Händler, der ein Gebrauchsgut von einem Kollegen, nämlich einem anderen Händler, oder von einem Privatmann ersteht. Schließlich wird ein solcher Schutz nicht für die "gebrauchten Sachen" gesetzlich geregelt, die "in einer öffentlichen Versteigerung verkauft werden, an der der Verbraucher persönlich teilnehmen kann". Die Beschränkung der langen Haftungsdaver auf Unternehmer-Verbraucher-Verkäufe macht das Anliegen der EU-Richtlinie, nämlich den Schutz des Endverbrauchers beim Kauf von Sachen für den Gebrauch, deutlich.

Nach der Neufassung des BGB erstrecken sich die Bestimmungen für den "Verbrauchsgüterkauf" auch auf Tiere, speziell auf Pferde. Das heißt, diese Bestimmungen betreffen unter anderem Tiere, weil die bisher im deutschen Recht (BGB $\S \S 481$ ss.) kodifizierten Sonderregelungen der Haftung für Mängel von (verkauftem) Vieh ersatzlos gestrichen wurden. Aus dem Gesetz verschwand speziell die (am 27. März 1899 erlassene) "Kaiserliche Verordnung", die die "Hauptmängel"(Rotz, Dummkoller, Dämpfigkeit, Kehlkopfpfeifen, Periodische Augenentzündung und Koppen) als solche beschrieb, für die ein Verkäufer - anders als bei den "Nebenmängeln" - 
unter anderem bei Pferden zu haften hatte, und die ferner die Frist, in der die Mängel sich zeigen mussten, auf 14 Tage festlegte. Ohne nähere Erklärung glich das neue Gesetz demnach die Tiere im Hinblick auf den Schutz des Verbrauchers den (leblosen) Verbrauchsgütern an, zum Beispiel den Automobilen, den Kühlschränken und den Bügeleisen. Dieser Schutz beinhaltet, wie gesagt, die Feststellung von (den Gebrauch der erworbenen Ware schmälernden oder behindernden) Mängeln, die Haftung des Verkäufers bei solchen Mängeln, die Daver der Haftung von zwei Jahren beziehungsweise von einem Jahr sowie die Umkehr der Beweislast im Fall von Mängeln, die sechs Monate nach der Übernahme des gekauften Gutes("Gefahrübergang") offenbar beziehungsweise angezeigt werden. Letzteres heißt, dass bei solchen Mängeln vermutet wird, sie hätten schon bei der Übernahme vorgelegen. Es heißt ferner: Stimmt der Verkäufer dieser Vermutung nicht zu, so hat er den Beweis dafür anzutreten, dass der Mangel bei der Übernahme noch nicht bestand. Von der genannten Vermutung wird allerdings nicht ausgegangen, wenn diese mit der Art der Sache oder der des Mangels unvereinbar ist. In diesem Sinne wird zum Beispiel nicht vermutet, eine Verletzung in einem akuten Stadium oder eine bestimmte Infektion (mit bekannter Inkubationszeit) habe bereits bei der Übernahme des Pferdes vor Wochen oder Monaten vorgelegen.

Die Umkehr der Beweislast, nämlich vom Käufer und Verbraucher auf den Verkäufer, die Dauer der Haftung sowie der Begriff des Mangels dürften in Zukunft die Zahl der Fälle mehren, in denen Käufer Haftungsansprüche gegen den Verkäufer anmelden. Zugleich wird, so lässt sich wie gesagt, weiter vermuten, beim Handel mit Pferden die Anzahl der Fälle steigen, in denen professionelle Verkäufer versuchen, Eigenschaften der von ihnen offerierten Pferde in Kaufuntersuchungen zu fixieren und die Tierärzte (im Rahmen eines Werkvertrages; Plewa 2002,286) derart in den Kaufprozess zu involvieren, dass sie (im Fall berechtigter Ansprüche durch den Käufer) zum Schadenersatz herangezogen werden können für die Folgen von unvollständigen oder unzutreffenden Aussagen, die aus Kunstfehlern und/oder aus mangelnder Sorgfalt resultieren. Weiter dürften die im Gesetz genannten Veriährungsfristen von zwei Jahren und von einem Jahr generell die Vorstellungen über Haftungszeiträume und das dementsprechende Verlangen von Käufern ändern. Aus diesem Grunde könnten ebenfalls Käufer häufiger als zuvor gegenüber (von ihnen bestellten) Gutachtern Schadenersatzansprüche anmelden. Käufer, die ihr Pferd von einem professionellen Händler erwerben, werden durch die neven Gesetze freilich weitgehend geschützt. Dies bedeutet unter anderem, dass sie nicht in dem Maße wie in der Vergangenheit einer Kaufuntersuchung bedürfen, in der die vom Verkäufer zugesicherten Eigenschaften vor der Nutzung des Pferdes überprüft beziehungsweise festgehalten werden.

Für den innerhalb von sechs Monaten nach der Übernahme ("Gefahrübergang") des Gebrauchsgutes im allgemeinen und des Pferdes im besonderen aufgetretenen Mangel wird wie gesagt vermutet, er habe bereits bei der Übernahme bestanden. Diese Frist von sechs Monaten ist bereits beträchtlich, das vor allem im Vergleich zu den 14 Tagen, innerhalb deren sich nach der Kaiserlichen Verordnung die Hauptmängel zu zeigen hatten, um den Anspruch auf die Wandlung des Kaufs nicht zu verlieren. An die zwei Wochen, in der sich ein Hauptmangel offenbaren musste, schloss sich nach altem
Recht eine Veriährungsfrist von sechs Wochen an. Im Vergleich zu diesen Zeiträumen ist ferner die Frist von zwei Jahren beziehungsweise von einem Jahr beträchtlich, bis zu der die Haftung für neue beziehungsweise für gebrauchte Verbrauchsgüter ausgedehnt wurde. Die Verjährungsfristen von 24 beziehungsweise 12 Monaten dürften für den veterinärmedizinischen Gutachter, wie gesagt, insbesondere dann relevant werden, wenn in späteren Untersuchungen Fehldiagnosen und/oder unvollständige Aussagen der (früheren) Kaufuntersuchung, zum Beispiel die unzutreffende Beurteilung von Röntgenbildern, aufgedeckt werden und diese Diagnosen sowie Aussagen die Kaufentscheidung des Verbrauchers mitbestimmt hatten. Angesichts des mit einem Gutachten verbundenen Risikos liegt für den Tierarzł ebenso wie für den Verkäufer der Versuch nahe, Tiere im allgemeinen und Pferde im besonderen möglichst häufig oder sogar generell als "nicht neu" oder als "gebraucht" zu qualifizieren und damit die Gewährsfrist des Verkäufers von zwei Jahren auf ein Jahr zurückzuführen, das heißt für den Tierarzt, das mit der Kaufuntersuchung verbundene Risiko zu reduzieren. Die Gefahr, zum Ersatz eines Schadens herangezogen zu werden, minimiert sich mit der Halbierung der Veriährungsfrist allerdings nicht einfach auf die Hälfte. Dies wäre nur dann der Fall, wenn die Ansprüche auf Schadenersatz sich im ersten und im zweiten Jahr nach der Übernahme eines Pferdes gleichmäßig verteilen würden, was wahrscheinlich aber nicht der Fall ist beziehungsweise sein wird. Die Verjährungsfristen von 12 beziehungsweise von 24 Monaten betreffen, wie gesagt, den Verbrauchsgüterkauf, nicht die Ansprüche, die aus dem Werkvertrag zwischen dem (als Gutachter tätigen) Tierarzł und seinem Auftraggeber resultieren.

Beträchtlich sind die genannten Fristen nicht nur im Hinblick auf die vom Gesetz als "Verbrauchsgüter" behandelten Tiere. Beträchtlich sind sie ebenfalls beim mangelhaften Kühlschrank und beim mangelhaften Staubsauger, bei denen bis zu sechs Monate nach dem Erwerb von einem mit diesen Geräten vereinbaren Mangel angenommen wird, er habe schon beim Erwerb bestanden, und für deren Gebrauchsfähigkeit der professionelle Verkäufer bis zu 24 beziehungsweise 12 Monate haften muss.

\section{Der Sache "entsprechend"}

Der Frage, inwieweit die für die Qualifizierung üblicher Verbrauchsgüter in bestimmter Hinsicht geeigneten Begriffe "neu" beziehungsweise "gebraucht" sich auf Tiere im allgemeinen und auf Pferde im besonderen übertragen lassen, soll hier unabhängig von den Interessen der Verkäufer, der Käufer und der mit Gutachten betrauten Tierärzte nachgegangen werden. Unter anderem ist zu erörtern, inwieweit das Gesetz Tiere mit den üblichen Verbrauchsgütern gleichsetzt, und zwar ohne deren spezifische, nämlich von denen der üblichen Verbrauchsgüter abweichende, Eigenschaften zu respektieren. Letzteres bedeutet nicht, die konforme Behandlung von Lebewesen und toten Sachen ethisch in Frage zu stellen und dem Gesetzgeber vorzuwerfen, er habe den inzwischen grundgesetzlich geschützten Anspruch der Tiere missachtet und diese Lebewesen in einer ethisch inakzeptablen Weise als Sachen behandelt. Laut § 90a des Bürgerlichen Gesetzbuches "sind" Tiere nämlich "keine Sachen". Sie "werden durch besondere Gesetze geschützt", und die "für Sachen geltenden Vorschriften" sind "entsprechend anzuwenden, soweit nicht etwas 
anderes bestimmt" ist. Nach diesen Sätzen kann man gegen das "Verbrauchsgüterkaufgesetz" vorbringen, es wende die für Sachen geltenden Vorschriften nicht "entsprechend", sondern unterschiedslos an.

Hier soll die Übertragung von Begriffen und Maßnahmen auf Pferde quasi "sach"logisch erörtert werden, nämlich die Übertragung auf Lebewesen, die anderen "Sach"gesetzlichkeiten gehorchen als die leblosen Verbrauchsgüter, zu deren Beschreibung und juristischen Behandlung die Begriffe und Maßnahmen geeignet sind und ursprünglich verwendet wurden. Die neuen Gesetze zum "Verbrausgüterkauf" untersuchen die Möglichkeiten und die Grenzen einer solchen Übertragung nicht. Sie unterstellen vielmehr, mit den für die Beschreibung eines bestimmten Zustandes von (leblosen) Verbrauchsgütern geeigneten Kategorien ließen sich auch bestimmte rechtsrelevante Qualitäten von Tieren zutreffend erfassen und in einer für die Rechtspraxis geeigneten Form behandeln.

\section{"Neu hergestellte" und "gebrauchte" Sachen}

Im hier angesprochenen Zusammenhang (BGB § 474 ss.) verwendet der Gesetzgeber den Begriff "neu", indem er von "neu hergestellten Sachen" sowie von "an einen Verbraucher verkaufte(n) neu hergestellte(n) Sachen" redet. Er spricht weiter von einer "gebrauchten Sache". Nach dem (insofern eindeutigen) Sinn des Textes stellt er die "gebrauchte" Sache also der "neven" gegenüber, nämlich die in einer öffentlichen Versteigerung verkaufte gebrauchte Sache und diejenige, für die er eine Veriährungszeit von einem Jahr bestimmt, und zwar im Gegensatz zur Verjährungszeit von zwei Jahren bei den "neuen" Sachen. Der Begriff "neu hergestellt" wird im Gesetz und in den Gesetzesmaterialien nicht erläutert.(Münchner Kommentar, p 2354)

Da weder der Begriff "gebraucht" noch der Begriff "neu" in eindeutiger Weise juristisch definiert wird, darf man davon ausgehen, dass der Gesetzgeber von der üblichen sprachlichen Verwendung der beiden Begriffe ausging, dass er ferner davon ausging, dass die umgangssprachliche Verwendung der Begriffe eindeutig ist. In der üblichen Sprache bedeutet das "Gebrauchen" einer Sache - nicht das "Brauchen" im Sinne des Benötigens - diese Sache zu nutzen, zu benutzen, sich die Sache zunutze zu machen, sie auszunutzen, sie in (einen bestimmten) Dienst zu stellen, sich ihrer zu bedienen, sie zu bedienen, sie anzuwenden, sie zu verwenden, sie zu verwerten, sie zu behandeln, sie zu betätigen, mit ihr umzugehen und auch sie zu manipulieren. In den juristischen Kommentaren heißt es, eine Sache sei gebraucht, wenn sie "in Benutzung genommen", beziehungsweise eine Sache sei dann "neu", wenn sie "noch nicht in ihren bestimmungsgemäßen Gebrauch genommen" worden sei. Der "bestimmungsgemäße" Gebrauch ist der dem Gebrauchsgut aufgrund seiner Fabrikation quasi immanente, auch der "gewöhnliche" oder der vertraglich fixierte. Der bestimmungsgemäße Gebrauch eines Bügeleisens zum Beispiel ist das Bügeln, nicht seine Nutzung als Briefbeschwerer oder als Dokument einer bestimmten ästhetischen Auffassung im Industriedesign einer bestimmten Epoche. Üblicherweise macht also erst der bestimmungsgemäße Gebrauch ein Verbrauchsgut zu einem "gebrauchten". Im Hinblick auf den "bestim- mungsgemäßen Gebrauch" wurde unter anderem darauf hingewiesen, dass eine Tages- beziehungsweise Formalzulassung einen Neuwagen nur dann zu einem Gebrauchtwagen mache, wenn "bereits eine Ingebrauchnahme zur Teilnahme am Straßenverkehr" vorliege.(Hoeren 2002,561) Letzteres bedeutet dann auch, dass die bestimmungsgemäß benutzten Sachen auch dann nicht mehr neu sind, wenn sie noch "wie neu" beziehungsweise noch im Zustand der "Neuwertigkeit" sind.

\section{Das "Neu"-Sein und das Gebrauchen}

Für den Zustand des "Neu"-Seins kennt die deutsche Sprache bezeichnenderweise kein eigenständiges Verbum, nämlich kein Verbum außer der verbalisierten Konstruktion "neusein". Dies ist symptomatisch, weil das "Neu-Sein" das "Behandelt-Werden" oder "Behandelt-worden-Sein" ausschließt beziehungsweise weil das "Neu-Sein" durch eine solche Behandlung aufgehoben würde. Und der Begriff "erneuern" bezeichnet eine Tätigkeit, bei der etwas "Gebrauchtes" (mehr oder minder weitgehend) wieder in den Zustand der "Neu-Wertigkeit" versetzt wird. Das "Erneuerte" wird allerdings nicht im eigentlichen Verständnis des Begriffs "neu", nämlich nicht "neu" im Sinne von "ungebraucht". Meist bezeichnet man mit dem Begriff "ernevern" die Tätigkeit, die eine Sache in einen Zustand versetzt, in dem sie weitgehend die funktionalen Eigenschaften aufweist, die für ihren NeuZustand charakteristisch sind, das heißt, in dem die Sache ähnlich wie im Neuzustand funktioniert. Dem "Neuen" im eigentlichen Sinne des Wortes ist noch nichts angetan, mit ihm ist noch nichts gemacht worden. Als Synonyma für den Begriff "neu" lassen sich folgerichtig nur (bestimmte Umstände ansprechende) Adjektiva benennen, nämlich "neugeboren", "nagelneu", "fabrikneu", "ungebraucht", "unberührt" oder "frisch". Das Verständnis des "Neven" als des "Fremden" oder des "Originellen" ist deutlich abzuheben vom Begriff des "Neuen" als des "Ungebrauchten". Die Tatsache, dass treffende Synonyme für den Begriff "neu" sich nur schwer oder gar nicht finden lassen, ist bezeichnend für den sprachlich eindeutigen Zustand des "Neu-Seins" als eines "(Noch-)Nicht-gebraucht-Seins", und zwar im Gegensatz zum "Gebraucht-Sein", nämlich zu den verschiedenen Modi des "Gebrauchens" und zu den diesen Modi entsprechenden Synonyma für das "Gebrauchen".

\section{Das Gebrauchen, das Gebrauchspotential und die Wertminderung}

Im üblichen Sprachgebrauch wird mit dem Begriff des "Gebraucht-Seins" in der Regel ein Zustand von geringerem "Wert" bezeichnet, nämlich ein Zustand, der bei Verbrauchsgütern besagt, dass das Gebrauchspotential der zur Diskussion stehenden Sache gemindert beziehungsweise partiell oder in bestimmten Hinsichten bereits "aufgebraucht" ist. In diesem Sinne wird der Begriff "gebraucht" auch vom Gesetz im Zusammenhang mit dem "Verbrauchsgüterkauf" verwendet, nämlich für eine "Sache", die nicht mehr "neu", das heißt deren Gebrauchspotential partiell reduziert ist, für deren Gebrauchsfähigkeit der Verkäufer daher auch nur noch eine kürzere Zeit zu haften hat, nämlich 12 Monate an Stelle der 24 Monate für die neven Sachen. Der Gesetzgebung lag 
wohl die übliche Vorstellung zugrunde, dass "Verbrauchsgütern" ein mehr oder minder großes "Gebrauchspotential" beziehungsweise eine mehr oder minder große "Nutzungskapazität" innewohnt, jedenfalls ein Potential, das sich durch den Gebrauch reduziert und das nach mehr oder minder intensivem oder extensivem Gebrauch "aufgebraucht" beziehungsweise "verbraucht" ist. Diese Minderung des Gebrauchspotentials wird mit anderen Worten als "üblicher Verschleiß" oder als "Abnutzung" verstanden und von einem speziellen (Funktions-)"Mangel" abgehoben, die Folge des Gebrauchs juristisch gleichwohl in einem größeren "Mängelrisiko" gesehen.(Hoeren 2002,559) Mängel betreffen sowohl vereinbarte und/oder (objektiv) erwartbare (Soll-)Beschaffenheiten als auch vereinbarte und/oder (objektiv) erwartbare Verwendungszwecke.

Der Gesetzgeber sprach bezeichnenderweise nicht nur vom "Gebrauchen", sondern auch vom "Verbrauchen", nämlich von "Verbrauchs"gütern. Letzterer Begriff geht über das "gebrauchen" insofern hinaus, als er eine Nutzung bis zur "Unbrauchbarkeit" impliziert. Der den Schutz des Verbrauchers verfolgende Gesetzgeber ging zudem von der üblichen Praxis aus, nämlich davon, dass die "gebrauchte", nämlich in ihrem Gebrauchspotential reduzierte und partiell der Unbrauchbarkeit angenäherte Sache, zu einem geringeren Preis als die neue gekauft wurde.

Das "Gebrauchen" von "Verbrauchsgütern" stellt also ein partielles "Verbrauchen" beziehungsweise das "Nutzen" ein partielles "Abnutzen", nämlich eine Wertminderung, dar. Das "Gebrauchen" verändert die Sache in eine bestimmte Richtung; sie nähert sie ein Stück weit der Unbrauchbarkeit an. Sie modifiziert das Gebrauchsgut nicht in die andere Richtung, ermöglicht nicht oder optimiert nicht seine Brauchbarkeit. Das Auto, das erst nach dem Einfahren voll belastet werden kann beziehungsweise voll belastet werden konnte, stellt diese Veränderung in eine Richtung nicht in Frage. Beim Kühlschrank, beim Bügeleisen, bei den Möbeln und bei der Kleidung ist die skizzierte Folge des "Gebrauchens" ebenfalls offenbar, bei den Antiquitäten nur auf den ersten Blick nicht. Die Patina letzterer zeugt nämlich von deren Alter und deren Echtheit. Fachleute unterscheiden deutlich zwischen einer solchen Patina und den (wertmindernden) "Gebrauchsspuren". Selbst die vom Butler eingetragenen Schuhe gewinnen nur für den Hausherrn an Wert. Nach allgemeinem Urteil sind sie "gebraucht" und in ihrem Nutzungspotential, damit auch in ihrem "Wert" beziehungsweise ihrer Verwertbarkeit, gemindert. Und die Sammler schätzen die "Gebrauchsspuren" der Kultgegenstände außereuropäischer Kulturen nicht als Aufwertung oder Mehrung der (ursprünglichen) Nutzungskapazität, sondern als Zeugnis der Authentizität dieser Objekte. In den Gesellschaften, die sich dieser Gegenstände im Kult "bedienten", gilt der Gebrauch und gelten insbesondere Gebrauchsspuren häufig als eine Wertminderung, die die weitere rituelle Verwendung solcher Gegenstände ausschließt.

Die Nutzung ist die bei Gebrauchs- und Verbrauchsgütern übliche Ursache der Wertminderung. Das Gebrauchspotential kann aber auch durch andere Einflüsse gemindert oder "unbrauchbar" werden. Häufig besprochene Beispiele für diese Zustandsveränderung sind die Automobile, die lange Zeit auf Halde standen oder die bei der Überführung zum
Händler starker Hagel traf. In diesen Fällen spricht man zwar nicht von (im üblichen Sinne) "gebrauchten" Fahrzeugen, von "neven" aber ebenfalls nicht (mehr). Die besonderen Einflüsse und die aus ihnen resultierende Wertminderung nimmt den Fahrzeugen auch juristisch ihren "Neu"-Zustand. Im Vergleich zur (üblichen oder bestimmungsgemäßen) Nutzung stellen die angesprochenen Einflüsse außergewöhnliche Ursachen der Minderung des Nutzungspotentials dar.

\section{Hergestellte und gewachsene Objekte}

Das im skizzierten Sinne als Wertminderung verstandene "Gebraucht-Sein" ist ein Zustand, der im üblichen Sprachgebrauch vom "Neu-Sein" abgehoben wird, und zwar als dessen Negation. Das "Gebraucht-Sein" wird im Denken und in der Sachbeurteilung dem - als "(Noch-)Nicht-gebrauchtSein" verstandenen - "Neu-Sein" gegenübergestellt. Der Begriff des "Gebraucht-Seins" impliziert demnach denklogisch den des "Neu-Seins" beziehungsweise der Zustand des "Gebraucht-Seins" sachlogisch den des "Neu-Seins".

Diese Feststellung leitet zu der Frage über, welche Objekte beziehungsweise welche Klasse von Objekten man als "neu" qualifiziert beziehungsweise nach üblichem Sprachverständnis so qualifizieren kann. Bei den zuvor genannten Automobilen, den Kühlschränken oder den Bügeleisen ist eine solche Eigenschaftsbestimmung unproblematisch. Häuser bezeichnet man ebenfalls als "neu", und zwar im Gegensatz zu denen, die bereits bewohnt, nämlich in der Weise des Bewohnens "gebraucht" und dabei auch mehr oder minder weitgehend "genutzt" sowie "abgenutzt" wurden.

Die als "neu" qualifizierten Gegenstände sind, so ergibt die Durchsicht weiterer Beispiele, solche, die vom Menschen für einen bestimmten Verwendungszweck hergestellt wurden, Gegenstände, deren Zustand nach der kompletten Herstellung als "fertig", vor der Nutzung als "neu" und nach dieser als (mehr oder minder intensiv) "gebraucht" beschrieben wird. Das Gesetz spricht, wie gesagt, von den "neu hergestellten Sachen". Die als "neu" bezeichneten Gegenstände wurden beziehungsweise werden also produziert, seltener als individuelle, meist in Serie. Auf die von Tieren "gemachten" "Objekte", zum Beispiel auf den "neuen" Gang des Maulwurfs oder die (nach dem Kehren) "neven" Pferdeäpfel auf der Stallgasse, soll hier nicht weiter eingegangen werden. Angemerkt sei freilich, dass in diesen umgangssprachlichen Qualifizierungen das "Neu-Sein" in einem anderen Sinne als bei den Kühlschränken gemeint ist, nämlich nicht als der dem "Gebrauchen" vorangehende und durch dieses aufgehobene Zustand.

Die für einen bestimmten Zweck dienlichen Gegenstände wurden, wie gesagt, (vom Menschen) für diese Verwendung hergestellt; das heißt auch, sie haben sich nicht ohne Zutun des (den Zweck planenden) Menschen entwickelt, sind nicht gewachsen; sie sind nämlich leblose Gegenstände, das heißt keine Gegenstände, die - unter gewissen fördernden Bedingungen - "von selbst" entstehen. Sogar den Zustand der vom Hobbygärtner mit Mühe gezüchteten Radieschen wird man nicht als "neu" bezeichnen und von einem Zustand des "Gebraucht-Seins" abheben. Und wenn man umgangssprachlich die "neven", nämlich "neu" gewachsenen, Radieschen von den "alten", nämlich schon früher gewachsenen, 
unterscheidet, dann hebt man den Begriff "neu" nicht vom Begriff "gebraucht" ab, sondern differenziert mit ihm zwischen "Neuem" und "Altem", bezeichnet derart also (nur) die zeitliche Folge(der Herstellung oder der Entstehung). Eine solche Gegenüberstellung von "neu" und "alt" kann unter anderem dazu dienen, Verbrauchsgüter zu qualifizieren; sie ist aber eindeutig von einer Gegenüberstellung von "neu" und "gebraucht" zu unterscheiden.

Die Gegenüberstellung von "neu" und "alt" impliziert anders als die Opposition von "neu" und "gebraucht" - nicht generell den größeren Wert des Neuen; mit ihr räumt man vielmehr bald die Attraktion des Neven, bald die des Alten ein. Vergleicht man zum Beispiel "alte" und "neue" Modelle bei einem bestimmten Autotyp, dann wird in der Regel dem neven Modell ein höherer Wert als dem alten zugeschrieben. Die Autofreaks und spezielle Kenner gewichten den Wert aber nicht selten umgekehrt. Noch höher ist der Wert des Alten im Vergleich zum Neuen in den Augen der Händler sowie der Sammler der "Antiquitäten" im engeren Sinne.

Ebenso wie man die "neuen" Radieschen nicht von "gebrauchten" abhebt, tut man dies nicht bei den neben den "alten" "neu" gepflanzten Eichen. Man spricht bei den "alten" Eichen nicht von "gebrauchten", obwohl die Bäume viele Jahrzehnte Schatten spendeten und obwohl mit ihren Früchten ganze Generationen von Schweinen gefüttert wurden. Wer die "neu" gepflanzten Eichen präziser charakterisiert, bezeichnet sie als "junge" Pflanzen. Er tut dies auch angesichts der Möglichkeit, älteren Gewächsen einen "neven", nämlich anderen, Standort zu geben.

Nur die temporale Folge hat man ebenfalls im Auge, wenn man unpräzise von "neven" Versuchstieren redet und damit eigentlich "neu" eingetroffene, "neu" angelieferte oder aus der eigenen Zucht ins Labor überführte meint. Hier wird die "neve" Lieferung einer früheren, nämlich einer "alten", gegenübergestellt. Charakterisiert man demgegenüber die Tiere selbst, dann werden diese unter anderem als "jung" oder "alt" beschrieben, nicht als "neu" oder "alt". Nennt jemand ein zuvor zu einem Versuch herangezogenes Tier als "gebraucht", dann dokumentiert er, so das verbreitete Urteil, den Umgang mit dem Tier als einer "Sache". Dem "gebrauchten" Versuchstier stellt der Inkriminierte meist nicht ein "neves", sondern ein "noch nicht in einen Versuch einbezogenes" oder auch ein "noch nicht gebrauchtes" gegenüber. Das Deutsche Tierschutzgesetz (§ 9,5 ss.) spricht vom "Verwenden" eines Versuchstieres. Nur in Ausnahmefällen bezeichnen Reiter ein "ausgedientes" Pferd als "verbraucht", zum Beispiel ein Spring- oder Jagdpferd nach zwölfiährigem Dienst oder ein Pferd, das ein Fiaker lange Jahre nutzte oder dessen ein Baver sich mehr als ein Jahrzehnt als Zugkraft bediente. Solche als "verbraucht" - nicht als "gebraucht" charakterisierten Pferde zeigen in der Regel "Verschleißerscheinungen" in der Form klinisch auffälliger Symptome. Als "neu" bezeichnet man Pferde selbst umgangssprachlich nicht. Kunstwerke im engeren Sinne werden ebenfalls nicht mit den Begriffen "neu" und "gebraucht" gekennzeichnet. Sie stellen keine "Gebrauchs"gegenstände im üblichen Sinne dar, wiewohl man das Ansehen dieser Werke und das Fasziniertwerden durch sie als einen Modus der Nutzung verstehen kann. Ein Ölgemälde Picassos zum Beispiel isł nach dem üblichen Sprach"gefühl" weder "neu" noch "gebraucht", auch dann nicht, wenn es entweder ständig unter einem dichten Rahmen geschütz† oder wenn es schon Jahre ohne Glasschułz hängt und häufig angesehen wird. Der Kunstmarkt umgeht die grundsätzliche Antwort auf die (auch für ihn akut gewordene) Frage nach "neu" oder "gebraucht" häufig dadurch, dass er die Werke nach einem Wechsel des Besitzers oder bei der Einlieferung in eine Auktion generell und pauschal als "gebraucht" klassifiziert. In manchen Fällen umgeht er die grundsätzliche Antwort neverdings auch dadurch, dass er dem Kunden pauschal eine Haftung von 24 Monaten einräumt. Kunsthandwerkliche Arbeiten sind meist ohne besondere Schwierigkeiten als "gebraucht" einzustufen, zum Beispiel die Meißen-Teller aus dem 18. Jahrhundert. Gegen die Zuordnung "alter Gemälde" und "antiker Kunstgegenstände" zu den "neu hergestellten Sachen" wird in der juristischen Argumentation vorgebracht, in dem Zeitraum, der seit der Herstellung dieser Gegenstände vergangen sei, seien diese "einem besonderen Sachmängelrisiko ausgesetzt" gewesen, zum Beispiel durch die Lagerung und durch den Wechsel des Eigentümers. Nach dieser Argumentation definiert das Fehlen eines "gebrauchs- und altersabhängigen Sachmängelrisikos" beziehungsweise das "Noch-nicht-in-bestimmungsgemäßenGebrauch-genommen-worden-Sein" generell die Qualifizierung von Gegenständen beziehungsweise "Sachen" als "neu hergestellt". Dieses Kriterium für die Abgrenzung führt juristische Kommentatoren unter anderem zu der Ansicht, selbst "auf natürliche Weise entstandene" Sachen, zum Beispiel Wein, Holz, Baumsetzlinge, Blumenstauden und auch Haustiere, könnten - im Gegensatz zum üblichen Sprachgebrauch - als "neu hergestellt" kategorisiert werden. Dabei erörtern die Kommentatoren allerdings nicht, inwieweit es sich bei den von ihnen genannten Beispielen um Verbrauchsgüter handelt, nämlich um gebrauchsfertige Güter im Sinne des Verbrauchsgüterkaufs. (Münchener Kommentar, p 2354 ss.)

Nach dem bisher Gesagten lässt sich das "Gebraucht-Sein" definieren, nämlich als die Aufhebung des "Neu-Zustandes" von (vom Menschen) für den Gebrauch gefertigten leblosen Objekten, und zwar die Aufhebung des "Neu-Zustandes" durch die Nutzung beziehungsweise als charakteristische Folge der Nutzung, nicht irgendeiner Nutzung, sondern in der Regel der bestimmungsgemäßen. Die Aufhebung des "NeuZustandes" besteht üblicherweise in einer mehr oder minder weitgehenden Veränderung der Objekte, nämlich in der üblichen Abnułzung beziehungsweise im üblichen Verschleiß. Die Veränderung schreitet bei anhaltender Nutzung in der Regel fort; sie reduziert - abhängig von ihrem Ausmaß - das Nutzungspotential, das durch die Herstellung im Objekt angelegt ist. Mit dem Zustand des "Gebraucht-Seins" verbindet man häufig freilich nicht nur die sachimmanenten und insofern unvermeidlichen Folgen der bestimmungsgemäßen Nutzung, sondern auch Veränderungen, die unter anderem aus einer unsachgemäßen Verwendung und/oder Bedienung resultieren und das Nutzungspotential über die übliche Abnutzung hinausgehend schmälern. Mit dem "Neu-Sein" bezeichnet man demgegenüber den Zustand eines hergestellten Objektes nach dessen Fertigstellung und vor dessen Gebrauch, also das Unbeeinträchtigt-Sein durch die üblichen Folgen einer bestimmungsgemäßen Nutzung oder durch außergewöhnliche Einflüsse, mit anderen Worten also das Ungeschmälert-Sein des Nutzungspotentials beziehungsweise des Wertes(aus der Sicht eines Käufers und/oder Konsumenten). 
Die derart als "gebraucht" oder "neu" definierten Waren für einen bestimmten Zweck schließen Qualitätsunterschiede nicht aus, zum Beispiel Unterschiede des Materials und seiner Verarbeitung bei den der Fortbewegung dienenden Autos, den dem Kühlen dienenden Schränken oder den dem Bügeln dienenden Eisen. Material und Verarbeitung dürfen allerdings ein bestimmtes Limit der Belastbarkeit (durch die Nutzung) nicht unterschreiten. Dieses Limit wird durch die Gebrauchsfähigkeit bei mehr oder minder intensiver und zeitlich mehr oder minder ausgedehnter Nutzung bestimmt. Die Gebrauchsfähigkeit hat der Verkäufer zu gewährleisten, allerdings nur eine Gebrauchsfähigkeit für den produktspezifischen Zweck, nicht die Gebrauchsfähigkeit bei zweckwidriger Anwendung, zum Beispiel nicht die Gebrauchsfähigkeit des Heimgarten-Rasentrimmers für das Abmähen einer großen Weide.

Die Überlegungen zur Verwendung des Begriffspaares "gebraucht - neu" machen deutlich, dass dieses - bei präzisem Einsatz der Sprache - nur zur Charakterisierung des Zustandes von (vom Menschen) für einen bestimmten Gebrauchszweck hergestellten Gegenständen verwendet wird und unter anderem nicht dazu dient, den Zustand von Pflanzen und Tieren im allgemeinen sowie den von Pferden im besonderen zu beschreiben. Diese sprachliche Praxis ist sachlich begründet, das heißt, der unterschiedliche Einsatz von Begriffen beruht auf Unterschieden in den Objekten, hier vor allem Unterschieden in den für einen bestimmten Zweck hergestellten leblosen Gebrauchsgegenständen einerseits und den aufgrund bio-logischer Dispositionen gewachsenen Pflanzen und Tieren andererseits.

\section{Die Problematik des gebrauchsfertigen "Neu"-Zustan- des eines Pferdes}

Nach den vorangegangenen sprachlichen Erörterungen sind jetzł die sachlichen Gründe für die mangelnde Eignung des Begriffspaares "gebraucht - neu" für die Beschreibung des Zustandes eines Pferdes aufzuzeigen. Dabei ist vor allem deutlich zu machen, dass die Anwendung des Begriffes "neu" auf ein Pferd noch problematischer ist als die des Begriffes "gebraucht".

Ungeeignet sind die Begriffe "gebraucht" und "neu" für die Beschreibung des Zustandes eines Pferdes, weil dieses nicht mit einem Material bestimmter Qualität und durch eine Verarbeitung bestimmter Qualität für einen bestimmten Zweck her- und fertiggestellt wird, das Lebewesen vielmehr aufgrund biologischer Gesetzlichkeiten entsteht und sich weiterentwikkelt. Das Pferd verhält sich nicht wie das mit technischen Materialien und nach technischen Normen hergestellte Gebrauchs- und Verbrauchsgut gemäß den Ideen seines Konstrukteurs, es entfaltet sich demgegenüber im Rahmen der dem Leben entsprechenden Abläufe. Dies betrifft seine Entstehung ebenso wie seine weitere Entwicklung, seine Unterhaltung ebenso wie seine Nutzung durch den Menschen. Ein Pferd lässt sich - trotz allen züchterischen Bemühens - nicht wie ein Gebrauchsgegenstand für einen bestimmten Zweck herstellen und nach dieser Produktion als "neues Gebrauchsgut" für diesen Zweck nutzen. Das Pferd kann auch nicht wie ein Gebrauchsgut fertiggestellt, als gebrauchsfertige Neuware in Erwartung eines Käufers eingelagert und nach dem Kauf durch den Konsumenten ohne weiteres Zutun genutzt werden. Zudem verläuft die "Abnutzung" beziehungsweise der "übliche Verschleiß" bei ihm anders als bei einem leblosen Verbrauchsgut.

Der gebrauchsfertige Neuzustand stellt eine integrale Eigenschaft der Gebrauchs- und Verbrauchsgüter dar, die der Gesetzgeber bei der Regelung des Verbrauchsgüterkaufs im Auge hatte, von der er nämlich das Gebraucht-Sein als alternative Eigenschaft abhob. Ein gebrauchsfertiger Neuzustand existiert beim Pferd jedoch nicht. Er existiert generell nicht bei einem Tier, bestenfalls angenähert bei einem nur undifferenziert dem Menschen dienenden, in erster Linie in seinem Dasein gesicherten oder für den Verzehr gezüchteten Tier, gewiss nicht bei einem differenziert vom homo sapiens genutzten.

Beim Hinweis auf die nicht gegebene Möglichkeit, ein Pferd für einen bestimmten Zweck anzu-fertigen, wird das züchterische Bemühen nicht ignoriert. In einem prinzipiell anderen Maße als die technische Bearbeitung eines leblosen Rohstoffes bleibt das züchterische Bemühen aber an biologische Abläufe und die ihnen entsprechenden Imponderabilien gebunden. Diese beginnen mit dem Eintreten der Trächtigkeit, für das der Züchter zwar vieles, aber nicht alles tun kann. Die Geburt eines gesunden Fohlens liegt ebenfalls nur begrenzt in der Hand des Züchters, ebenfalls die Eignung des unversehrt herangewachsenen Pferdes für das Reiten im allgemeinen und für die Nutzung im Springparcours oder auf dem Dressurviereck im besonderen.

Pferde werden mit unterschiedlichen Anlagen für die verschiedenen Verwendungszwecke geboren, insbesondere für die spezifische Verwendung beziehungsweise die Verwendung unter hohen Leistungsanforderungen unterschiedlichen Anlagen. Ihnen eignen quasi unterschiedliche Materialqualitäten und Funktionsdispositionen; sie entstehen nicht wie die Mehrzahl der Verbrauchsgüter - sieht man von den sogenannten "Montags"produkten ab - in einer Serie gleicher Objekte mit gleicher Eignung für einen bestimmten Zweck.

Auf die Entfaltung der Frucht mit unterschiedlichen Anlagen gewinnen die Konstitution sowie die Kondition der Stute Einfluss, ferner die Fütterung, die Haltung, die veterinärmedizinischen Maßnahmen sowie die weiteren Umstände in der Zeit der Trächtigkeit. Die pränatalen Faktoren werden im Fohlen mehr oder minder manifest, später auch in der Nutzbarkeit des herangewachsenen Pferdes. Derartige Einflüsse lassen sich schwieriger bestimmen, scheinen aber doch weiter zu reichen als diejenigen, die das Material der meisten Gebrauchsgüter vor oder bei seiner Verarbeitung erfahren hat und die die Funktionsfähigkeit der Gebrauchsgüter mitbestimmen.

Weit reicht ferner der Einfluss des menschlichen Handelns bei der Geburt und nach der Geburt des Fohlens, der Einfluss durch den Umgang mit dem "Neu"geborenen und dem jungen Fohlen, auch der Einfluss durch die Haltung und Fütterung der Stute, der Einfluss im Rahmen der "normalen" Haltung und Fütterung, aber auch der Einfluss im Fall gesundheitlicher Komplikationen oder im Fall von Verhaltensstörungen, der Einfluss durch mehr oder minder fachgerechtes menschliches Engagement und auch der Einfluss durch das Unterlassen einer bestimmten Tätigkeit. Über die Bedeutung 
solcher Einflüsse und Einwirkungen wusste man schon in früheren Jahrhunderten; heute weiß man vor allem mehr über ihre bio-logische Wirkweise und ihre bio-logische Bedeutung, ferner über ihre Relevanz für die spätere Biographie des Pferdes. Diese Einflüsse - zum Beispiel die Begegnung mit Artgleichen, speziell die mit der Mutter, und die mit Artfremden, speziell mit dem Menschen ("Prägung"), oder der Stoffwechsel vor der Geburt und der des "Neu"geborenen - sind ungleich komplexer als die Einflüsse, denen das Material der Verbrauchsgüter vor seiner Verarbeitung ausgeliefert ist. Diese gravierenden Unterschiede verbieten es, die Einflüsse auf das Lebewesen und deren allgemeines Lebensrisiko den Einflüssen auf das Material der Gebrauchsgüter gleichzusetzen. Zudem lässt sich das Material für die Verbrauchsgüter durch eine an der Gebrauchsfähigkeit orientierte Norm im Hinblick auf seine Eignung beurteilen und selektieren, was bei den Pferden - bis auf die (natürliche und die durch das Wirken des Menschen erreichte) Aussonderung der in ihrer Gesundheit erheblich angegriffenen Individuen - so nicht geschieht. Das Absetzen des Fohlens, seine Eingliederung in eine Herde Gleichaltriger und seine Aufzucht unter mehr oder minder zureichenden biotopischen und sozialen Bedingungen, die Gewöhnung des heranwachsenden Fohlens an den menschlichen Zugriff, das Aufstallen des jungen Pferdes, die ersten Ausbildungsschritte an der Longe, das Auflegen des Sattels, das Einreiten und die Grundausbildung unter dem Sattel lassen sich als markante Maßnahmen beziehungsweise als markante Stadien in der fortlaufenden Einwirkung auf das Pferd verstehen. Weitere Stadien und/oder Zäsuren in dieser anhaltenden Einwirkung bilden die der Grundausbildung folgende Spezialausbildung, der Übergang des Pferdes vom Züchter zum Händler oder zum "Verbraucher", ferner der Übergang aus der Hand des Ausbilders in die des "Verbrauchers", der Einsatz des Pferdes im Leistungssport durch den "Verbraucher" oder der Einsatz des Pferdes durch den "Verbraucher" im sogenannten Freizeitsport, letzterer Einsatz gleich nach der Grundausbildung oder nach einer mehr oder minder erfolgreichen Karriere im Leistungssport.

\section{Die natürlichen Einflüsse sowie die Einwirkungen des Menschen und das Nutzungspotential des Pferdes}

Die mit den genannten Maßnahmen, Stadien und Zäsuren verdeutlichte fortlaufende Einwirkung des Menschen auf das Pferd schlägt sich im Zusammenhang mit den Folgen der zuvor genannten natürlichen Einflüsse im Gefüge der Eigenschaften und der Bereitschaften des Pferdes nieder; speziell schlägt diese Einwirkung sich in der Nutzbarkeit des Pferdes nieder, in der allgemeinen Einsetzbarkeit unter dem Sattel und in der Eignung für besondere Aufgaben und besondere Anforderungen, also in seinem Nutzungspotential. Die diversen Modi der menschlichen Einwirkung auf das Pferd und speziell die diversen Maßnahmen der frühen und der weiterführenden Ausbildung des jungen Pferdes sind, wie vor allem immer wieder auftretende unfallbedingte Verletzungen und sukzessive sich entwickelnde Schäden offensichtlich dokumentieren, mit einem nicht zu vernachlässigende Gesundheitsrisiko verbunden. Dieses ist bei fachlich inkompetentem Umgang mit dem Pferd besonders hoch. Zudem verbindet sich das durch die menschlichen Einwirkungen - und die menschlichen Unterlassungen - bedingte spezielle Gesundheitsrisiko mit dem allgemeinen Gesundheitsrisiko, nämlich dem der intrauterinen Existenz der Frucht, der Geburt und des weiteren Aufwachsens. Das Aufwachsen eines Lebewesens im Allgemeinen und das des Pferdes im besonderen ist, wie gesagt, unter anderem mit Verletzungen und Schäden verbunden, die das Nutzungspotential des Tieres mehr oder minder beeinträchtigen. Die Vorstellung, die Lebewesen würden sich bis zur Nutzung durch den Menschen heil und unversehrt entwickeln, fördert zwar das illusionäre Weltbild mancher Menschen; sie entspricht jedoch nicht der Wirklichkeit. Das intensive Plädoyer für das Aufwachsen der Fohlen in der Herde Gleichaltriger auf großen Arealen zum Beispiel sollte die trotz beträchtlicher menschlicher Sorgfalt auftretenden Verletzungen und Schäden beim Weidegang nicht übersehen lassen. Verletzungen und Schäden stellen Begleiterscheinungen des Lebens dar. "Leben" isł ein Synonym für Veränderung, nämlich für Modifikationen, die die Ei- und die Samenzelle ebenso betreffen wie das alternde Individuum und die im Hinblick auf die Möglichkeiten der Nutzung des Individuums durch den Menschen generell meist ambivalent sind. Insofern unterscheiden das Leben generell und das Heranwachsen der Individuen speziell sich grundlegend von der Herstellung, von der Lagerung und vom Gebrauch der (leblosen) Verbrauchsgüter. Insbesondere existiert bei Lebewesen kein statischer Zustand, der dem der Verbrauchsgüter nach der Fertigung und vor dem Gebrauch entspricht.

Die diversen üblichen und die außergewöhnlichen "natürlichen" Ereignisse sowie die diversen Modi der menschlichen Einwirkung auf das Tier schlagen sich, wie gesagt, in dessen Nutzungspotential nieder. Die Auswirkungen auf das Nutzungspotential lassen sich als quantitative oder als qualitative begreifen. Häufig zeigt sich die Relevanz dieser Auswirkungen erst im Rahmen der Ausbildung und im Rahmen des bestimmungsgemäßen Gebrauchs des adulten Pferdes. Die Auswirkungen der mehr oder minder natürlichen Einflüsse sowie die der menschlichen Einwirkungen reichen häufig weiter, sind aber nicht ähnlich offenbar wie die Mängel, die bei noch nicht bestimmungsgemäß genutzten Autos aus ihrem langen Stehen auf Halde resultieren und die diesen Autos in juristischer Hinsicht ihren "Neu"-Zustand nehmen. Jedenfalls sind die natürlichen Einflüsse inklusive des allgemeinen Gesundheitsrisikos und die menschlichen Einwirkungen auf ein Lebewesen nicht dem unter normalen Umständen weitgehend stabilen Zustand eines leblosen Gebrauchsgegenstandes nach seiner Produktion und vor seiner Indienstnahme gleichzusetzen. Gerade in Entsprechung zu den Einflüssen und Einwirkungen auf leblose Verbrauchsgüter muss man die (nicht vom Menschen intendierten) natürlichen Einflüsse sowie die vom Menschen beabsichtigten Einwirkungen auf das noch nicht bestimmungsgemäß genutzte Pferd als eine Art des "Gebrauchens" beziehungsweise als Prozesse mit Folgen auffassen, die denen des (bestimmungsgemäßen) Gebrauchs ähnlich sind. Orientiert man sich am Beispiel des verwitterten Lacks der Autos, so muss man selbst das wachsende Leben als einen Prozess verstehen, der nicht nur in der Fertigung und in der Politur, sondern zugleich auch in der Verwitterung besteht. Diese unter anderem einzelne Zellen betreffenden biologischen Gesetzlichkeiten lassen folgerichtig die häufig genannten Zeitpunkte in Frage stellen, ab wann ein Pferd als "gebraucht" zu gelten habe: Bald setz† man den Übergang des Pferdes vom Züchter an den "Verbraucher" als einen solchen Zeitpunkt an, bald das Einreiten, bald das Absetzen des Fohlens, bald seine Geburt (Brückner und Böhme 2002). 
Derartige Zeitpunkte können sich leicht verschieben, wenn man ihrer Bestimmung die unterschiedlichen Nutzungsweisen von Pferden zugrundelegt und die "bestimmungsgemäße" Nutzung als den "eigentlichen" Anfang des "Gebrauchens" versteht. Bei dem für den Verzehr gezüchteten Fohlen zum Beispiel beginnt die "eigentliche" Nutzung in einer anderen Altersstufe als beim Traber oder beim Galopper, bei den Rennpferden in einer anderen Altersstufe als bei den gleich nach der Grundausbildung als Freizeitpferd eingesetzten Warmblütern, beim Jagdpferd sowie beim Turnierpferd für die Anfängerklasse in einem anderen Stadium als beim GrandPrix-Pferd, das auf der Auktion als Aspirant "für höchste Ansprüche" erworben und mit dem Ziel der Grand-Prix-Reife in die Hände des professionellen Ausbilders gegeben wird. Versteht man letztere Ausbildung als den Prozess der Endfertigung eines Grand-Prix-Pferdes, dann beginnt dessen projektierte Nutzung erst mit dem Einsatz in der Prüfung der schweren Klasse, also im Alter von acht oder neun Jahren, nämlich in einem Lebensabschnitt, in dem die Rennpferde ihren Dienst auf der Bahn in der Regel lange hinter sich haben. Auf den ersten Blick macht es zwar Sinn, das 4-jährige Pferd nicht im gleichen Maße oder nicht in gleicher Weise als "gebraucht" einzustufen wie das 14-jährige, das ein knappes Jahrzehnt über Weiden und Felder hinter den Hunden galoppierte. Und der angerittene Vieriährige ist in anderer Weise als das erstmals ans Euter seiner Mutter geführte Saugfohlen "gebraucht". Mit solchen Beispielen lassen sich die verschiedenen Zeitpunkte, ab denen man ein Pferd als "gebraucht" einstufen könnte, freilich auch problematisieren und relativieren. Das heißt: Über die Argumentation gegen einen und für einen anderen dieser Zeitpunkte ist das Problem des "Gebraucht"-Seins des Pferdes nicht grundsätzlich lösen.

\section{Verbrauchsgüter und Rohmaterial}

Den Ansatzpunkt für die prinzipielle Beantwortung der anstehenden Frage stellt demgegenüber der mehrfach schon angesprochene Sinn des Gesetzes über den Verbrauchsgüterkauf dar, speziell die Rede von den "gebrauchten Sachen" (§ 475,2), die, den übrigen Formulierungen und dem Sinn des Gesetzes entsprechend, als "gebrauchte" "Verbrauchsgüter" zu verstehen sind. Den "gebrauchten" "Verbrauchsgütern" werden, wie zuvor erörtert, nicht-gebrauchte, nämlich "an einen Verbraucher verkaufte neu hergestellte Sachen", gegenübergestellt. Gemeint sind in dieser Gegenüberstellung demnach nicht irgendwelche neven "Sachen", sondern "Verbrauchsgüter", also zum Verbrauch geeignete Güter, das heißt gebrauchsfertige Güter, nicht das Rohmaterial, aus dem sich gebrauchsfertige Güter herstellen lassen. Behandelt wird im Gesetz der Verkauf an einen "Verbraucher", nicht der an einen Hersteller. Die Haftung für die Brauchbarkeit von Verbrauchsgütern ist insofern nicht mit der für Rohmaterial zu identifizieren; erstere schließt die bis zur Brauchbarkeit reichende Fertigung ein, und zwar eine Fertigung, die nicht zwingend, sondern nur bei materialgerechtem Vorgehen zur Brauchbarkeit führt. Das mit der Fertigung verbundene Risiko hat - im Sinne des Verbraucherschutzes - der Verkäufer beziehungsweise der Produzent zu tragen, der "Verbraucher" zahlt für ein gebrauchsfertiges Gut und er hat Anspruch auf die Lieferung eines gebrauchsfertigen Gutes, nämlich eines Gutes, das ohne weitere Fertigungsmaßnahmen in (den bestimmungsgemäßen) Gebrauch genommen werden kann.
In diesem Sinne wurde in anderem Zusammenhang betont, bei "als neu verkauften Sachen" dürfe "auf deren volle Gebrauchstauglichkeit vertraut werden". (Wolf et al. 1999, 1242 s.) Dies gilt auch für die Güter, die der Verbraucher wie zum Beispiel das im Baumarkt gekaufte Regal - selbst zusammensetzen muss, die er in der Regel aber auch kostengünstiger erwirbt als diejenigen, die der Verkäufer im Hause des Verbrauchers zusammenschraubt.

Selbst wenn man das Fohlen im Vergleich zum 14-jährigen Jagdpferd als "neu" kategorisieren würde, müsste man von diesem Fohlen doch feststellen, dass ihm - in anderer Weise als dem noch nicht zusammengesetzten Regal - der im Gesetz unterstellte Zustand der Gebrauchsfertigkeit nicht eignet. Dies gilt nicht minder für den Vieriährigen, den man als Pferd für höchste Ansprüche mit dem Ziel des Einsatzes auf dem Grand-Prix-Niveau auf der Auktion erwirbt. Nach dem Sinn des Gesetzes sind das Fohlen ebenso wie die Remonte quasi Rohmaterial, nämlich Pferde, die erst durch die - als eine Art "Fertigung" zu verstehende - Ausbildung den Status der (bestimmungsgemäßen) Gebrauchsfertigkeit erreichen. Das Aufwachsen beziehungsweise die Ausbildung stellen nicht den bestimmungsgemäßen Gebrauch dieser Pferde im Sinne des Gesetzes dar. Dieser besteht vielmehr im Einsatz unter dem Sattel oder vor dem Wagen. Das Aufwachsen und die Ausbildung entsprechen der Bearbeitung des Rohmaterials und den Fertigungsprozessen bei den üblichen Gebrauchsgütern. In diesem Sinne ist es bezeichnend, dass unbebaute Grundstücke juristisch nicht als "neu hergestellt" verstanden werden; ihnen fehlt nämlich, so die juristische Argumentation, "das Element der abgeschlossenen Vervollkommnung, das bei neu hergestellten Sachen gerade die Erwartung eines besonders geringen Sachmängelrisikos begründe". (Münchener Kommentar, p 2355) Diese "abgeschlossene Vervollkommnung" (als Gebrauchsgut) fehlt ebenfalls dem Reit- sowie dem Fahrpferd vor der speziellen Ausbildung. Das nur zum Zweck der Aufzucht erworbene Fohlen stellt in diesem Sinne ebenfalls kein gebrauchsfertiges "Verbrauchsgut" dar, nicht anders die Ei- und die Samenzelle sowie deren Verbindung. Ihr bestimmungsgemäßer Zweck ist es gerade, die noch ausstehende "Vervollkommnung" zu erreichen. Ihr Zweck besteht nicht darin, im Gebrauch "verbraucht" zu werden. Sie sollen vielmehr erhalten und in ihrer Potenz entfaltet werden, möglichst wenig gezeichnet von den belastenden natürlichen Einflüssen und den belastenden menschlichen Einwirkungen beziehungsweise möglichst wenig beeinträchtigt in den Eigenschaften, die für die Entwicklung und den späteren bestimmungsgemäßen Gebrauch eines Individuums relevant sind.

Das Gesetz befasst sich nicht mit dem Verkauf und der Lieferung von Rohmaterial (an einen Produzenten). Es verpflichtet den Lieferanten von Rohmaterial nicht in dem Maße zur Haftung, das für die Veräußerung eines (aus dem Rohmaterial hergestellten) gebrauchsfertigen Gutes vorgesehen ist. Das Gesetz unterstellt, dass der Verbraucher den Preis für ein gebrauchsfertiges Gut gezahlt hat, nicht den (geringeren) Preis für Rohmaterial. Dieser Exkurs über das im Gesetz nicht genannte Rohmaterial ist hier erforderlich, um die Gemeinsamkeit und die Differenz zwischen dem noch nicht "fertigen" Pferd und dem gebrauchsfertigen "Verbrauchsgut" deutlich zu machen und um ferner zu belegen, dass ein - dem der neven gebrauchsfertigen Verbrauchsgüter korrespondieren- 
der - Zustand des "Neu-Seins" und der gleichzeitigen "Gebrauchsfertigkeit" beim Pferd nicht existiert. Es kommt hier also auf die Koinzidenz von "Neu-Sein" und "Gebrauchsfertigkeit" an.

Ausnahmen bilden in dieser Hinsicht allerdings, wie bereits angesprochen, Pferde, die nicht differenziert genutzt und die mit dem Erreichen eines bestimmten Alters automatisch nämlich aufgrund des natürlichen Wachstumsprozesses und ohne differenzierte, mit Risiken und Einbußen der Nutzungskapazität verbundene Ausbildung - gebrauchsfertig werden, also vor allem die zum Verzehr gezüchteten und die ausschließlich als Kumpan (für Mensch oder Pferd) oder die ausschließlich als "Rasenmäher" dienenden Pferde. Freilich ist selbst für die zum Verzehr gezüchteten Fohlen nicht a limine auszuschließen, dass die nicht beabsichtigten Einflüsse und die intendierten Einwirkungen, denen die Tiere vor dem Schlachten ausgesetzt sind, zum Beispiel Stress, sich in der Fleischqualität niederschlagen und insofern als eine Modalität des "Gebrauchens" angesehen werden können. Mit dem ersten bezweckten und insofern eigentlichen "Gebrauch", nämlich der Schlachtung, isł das als Nahrungsmittel gezüchtete Pferd allerdings definitiv "verbraucht". Bei dem als artgleicher Kumpan oder als "Rasenmäher" eingesetzten Pferd stellt sich die Gebrauchsfertigkeit ebenfalls mit einem gewissen Alter ohne differenzierte Ausbildung ein. Bei solchen Pferden ist die Zuordnung zu den "gebrauchten" oder den "neuen" Verbrauchsgütern allerdings noch etwas problematischer als bei den für den Verzehr gezüchteten Tieren. Man könnte sich beim Sozialpartner- und beim Rasenmäher-Pferd über die Extreme pragmatischen Lösungen annähern, nämlich über das eine Extrem des wahrscheinlich als "neu" eingestuften jungen und gesunden Pferdes und das andere Extrem des alten und gesundheitlich beeinträchtigten Pferdes, auch über das eine Extrem des zwar unerfahrenen, aber unerschütterlichen Pferdes und das andere des aufgrund inkompetenter Behandlung zur Panik neigenden Tieres. Solche pragmatischen Annäherungen kommen allerdings nur in Frage, wenn man die hier vertretene grundsätzliche Lösung des Problems verwirft, nämlich bereits die dem bestimmungsgemäßen Gebrauch vorangehenden üblichen und außergewöhnlichen Einflüsse sowie die dem bestimmungsgemäßen Gebrauch vorangehenden diversen menschlichen Einwirkungen als Faktoren zu verstehen, die Folgen für das Lebewesen haben und einen potentiellen "Neu"-Zustand aufheben. Man darf freilich vermuten, daß weder die zum Verzehr gezüchteten noch die als Sozialpartner, noch die als Rasenmäher eingesetzten Pferde in der vorliegenden Hinsicht in der Rechtsprechung eine große Bedeutung gewinnen werden.

Die Nutzung von Pferden als Sozialpartner, als Rasenmäher oder als Nahrung ist - im Vergleich zur Nutzung unter dem Sattel oder im Zug - ähnlich undifferenziert wie die von Forellen und die übliche von Hunden. Auf diese Tiere wird hier hingewiesen, weil in einem Urteil aus dem Jahr 1985 Forellen, geliefert für eine Zucht- und Anglersportanlage, und in einem Urteil aus dem Jahre 1989 ein neun Wochen alter Hundewelpe den "neu hergestellten Sachen" zugeordnet worden sind. Insbesondere das Urteil über den Welpen begründete man mit dem Argument, der junge Hunde sei bisher "nur mit dem in seiner Existenz(Beschaffenheit) wurzelnden Lebensoder Gesundheitsrisiko behaftet" gewesen, "nicht aber mit dem typischerweise durch Gebrauch entstehenden". Man schätzte das Lebensrisiko des jungen Hundes in den zur Diskussion stehenden ersten neun Wochen als gering ein; "andere Umwelteinflüsse" hätten "noch keine allzu große Rolle gespielt" (Neve Juristische Wochenschrift-R R 1986, 52 ss.; Neue Juristische Wochenschrift 1990, 916)

Das Forellen- und das Welpenurteil ist meines Erachtens selbst auf undifferenziert genutzte Pferde nicht zu übertragen: Die Forellen erreichten wahrscheinlich schon ihre Gebrauchsfertigkeit, möglicherweise auch der Welpe, das heißt, dieser, wenn er ausschließlich als Sozialpartner gekauft wurde, jedoch nicht, sofern sein bestimmungsgemäßer Gebrauch die Funktion des Schutzhundes war. Die Forellen und die Welpen waren freilich - wie es die Pferde weiterhin sind - frühen natürlichen Einflüssen und menschlichen Einwirkungen ausgesetzt, die sich in den Individuen niederschlugen und von denen man annehmen kann, dass sie einen potentiellen "Neu"-Zustand aufhoben. Sofern die Forellen und der Welpe noch nicht gebrauchsfertig waren, fallen sie schon deshalb nicht unter die neven gebrauchsfertigen Güter und die frühen natürlichen Einflüsse inklusive des allgemeinen Gesundheitsrisikos sowie die menschlichen Einwirkungen gehören zum "Fertigungsprozess". Auch in diesem Fall verbietet es sich, wie gesagt, die frühen natürlichen Einflüsse, die frühen menschlichen Einwirkungen sowie das allgemeine Gesundheitsrisiko des jungen Tieres den Einflüssen gleichzusetzen, die leblose Gebrauchsgüter nach ihrer Fertigung und vor ihrem Gebrauch treffen. Geht man davon aus, dass die Forellen und der Welpe bereits gebrauchsfertig waren, dann stellen die natürlichen Einflüsse inklusive des allgemeinen Gesundheitsrisikos und die menschlichen Einwirkungen aufgrund ihrer unter Umständen beträchtlichen Auswirkungen Modi des Gebrauchs dar. In jedem Fall verändern diese Auswirkungen die "Sache". Die natürlichen Einflüsse und die menschlichen Einwirkungen beziehungsweise das allgemeine Gesundheitsrisiko sind daher nicht mit den (in der Regel minimalen und daher vernachlässigbaren) Einflüssen zu identifizieren, die die leblosen Verbrauchsgüter bei ordnungsgemäßer Lagerung nach ihrer Fertigung und vor ihrem Gebrauch erfahren.

Das Urteil über die Forellen und das über den Welpen wurden nicht im Rahmen des neven Verbrauchsgüterkaufrechts gefällt, sondern im Rahmen des alten Rechts im Hinblick auf die Anwendung des $\S 11$ Nr.10 des Gesetzes zur Regelung des Rechts der Allgemeinen Geschäftsbedingungen (AGBG). Dass diese Urteile im Rahmen des Gebrauchsgüterverkaufs in gleicher Weise gefällt worden wären und dass sie in diesem Rahmen Bestand haben und als generelle Richtlinien für die juristische Behandlung von (differenziert oder undifferenziert genutzten) Tieren dienen können, darf man mit Nachdruck bezweifeln. Und: Selbst in ihrem besonderen Zusammenhang ignorieren die beiden Urteile die Spezifität der stets von Einflüssen der Umwelt abhängigen Lebensprozesse einerseits und der (vergleichsweise weitgehenden) Stabilität lebloser Objekte andererseits. Bezeichnenderweise war es bereits vor der Einführung des Verbrauchsgüterkaufrechts umstritten, ob Tiere den "neu hergestellten Sachen" subsumiert werden können. Palandt zum Beispiel lehnte im Rahmen seines Kommentars zum AGBG §11 Nr. 10 eine solche Qualifizierung für Tiere ab, akzeptierte sie aber für pflanzliche Produkte, zum Beispiel für Gemüse und Obst.

Anders als bei den undifferenziert genutzten Tieren im allgemeinen und den undifferenziert genutzten Pferden im besonderen entsteht die Gebrauchsfertigkeit bei den zum Reiten 
und/oder Fahren dienenden Pferden, wie gesagt, nicht automatisch im Verlaufe des (natürlichen) Heranwachsens. Sie wird erst und nur aufgrund besonderen Zutuns des Menschen erreicht. Dieses Zutun besteht in üblichen Maßnahmen der Haltung, der Fütterung und der Pflege, auch in Maßnahmen der medizinischen Vorsorge und der Therapie, vor allem aber in der allgemeinen sowie der speziellen Ausbildung. Diese Maßnahmen gehen, wie gesagt, weit über das Wachsen-Lassen hinaus, sie sind mit Risiken verbunden, die das übliche Gesundheitsrisiko übersteigen und die einen beträchtlichen Einfluss auf die spätere Gebrauchsfähigkeit gewinnen. Insofern muss man diese Maßnahmen als eine Art von "Gebrauch" verstehen, und zwar nicht als den bestimmungsgemäßen Gebrauch, aber als einen "Gebrauch", der nicht selten auch zum "Verbrauch" führt. Letzteren belegt die Rate der Pferde, die von der Konzeption bis zur projektierten Verwendung ihre Einsatzfähigkeit verlieren, sei es durch Krankheit oder Tod, durch sukzessiv sich entwickelnde Schäden oder durch unfallbedingte Verletzungen, durch zu geringe Belastungsresistenz oder durch zu hohe Trainingsanforderungen, durch mangelnde Leistungsbereitschaft oder aufgrund anderer Verläufe. Selbst an den "Verbrauch" in der intrauterinen Phase der Frucht ist hier zu denken, zum Beispiel an Resorptionen durch die Überfütterung der Stute oder durch deren Infektion aufgrund mangelnder Hygiene. Die Überfütterung und die mangelnde Hygiene sind menschliche Maßnahmen, die sich auf die Frucht auswirken, und zwar Maßnahmen, bei denen die Frucht "gebraucht" und möglicherweise auch "verbraucht" wird. In der Genetik spricht man bezeichnenderweise davon, dass eine bemerkenswerte Anzahl von Ei- und Samenzellen sowie eine bemerkenswerte Anzahl von Konzeptionen eingesetzt, genutzt oder "verbraucht" wird, um ein gesundes Individuum zu gebären. In anderer Weise "gebraucht" man das Pferd, wie gesagt, bei seiner Ausbildung für seinen eigentlichen Verwendungszweck, vor allem bei der über das Anreiten hinausgehenden Schulung, speziell bei der Schulung für hohe Anforderungen. Im Rennsport ist die Spanne zwischen dem Trainingsbeginn und dem projektierten Derby-Start besonders riskant, das heißt, in dieser Phase wird ein bemerkenswerter Anteil der Pferde - aus verschiedenen Gründen - unfähig für den bestimmungsgemäßen Einsatz beziehungsweise "verbraucht".

In den angesprochenen Pflege- und Ausbildungsmaßnahmen eine Art von "Gebrauch" zu sehen, widerspricht nicht der Feststellung, der Zustand des "Neu-Seins" und der gleichzeitigen "Gebrauchsfertigkeit" existiere beim Pferd nicht. Der Begriff "Gebrauch" wird nämlich in beiden Fällen in unterschiedlicher Bedeutung verwendet, nämlich im einen Fall allgemein im Sinne einer mit Folgen für das Nutzungspotential verbundenen Einwirkung und im anderen als der bestimmungsgemäße Gebrauch(unter dem Sattel und/oder vor dem Wagen). Die Ausbildung des Reitpferdes ist, wie gesagt, nur über das Reiten möglich, allerdings über ein Reiten, das in anderer Weise verläuft als das Reiten, das als der eigentliche Verwendungszweck des Pferdes vorgesehen ist. Bezeichnenderweise wird die zur Gebrauchsfertigkeit führende Ausbildung häufig von Bereitern, also von professionellen Ausbildern, geleistet. Im gleichen Sinne werden Rennpferde auf der Rennbahn unter dem Sattel oder vor dem Sulky für ihre spätere Verwendung von professionellen Trainern geschult, und zwar im "Laufen", aber in einem Laufen, das sich im einzelnen anders vollzieht als der spätere Einsatz im Wettkampf.
Das "Reiten" und das "Laufen" auf der Rennbahn stellen also Oberbegriffe dar. Die durch das "Reiten" beziehungsweise das "Laufen" für ihre spätere Zweckbestimmung geschulten Pferde sind nur für diese Schulung "gebrauchsfertig", nicht aber für ihre projektierte, nämlich die "bestimmungsgemäBe", Verwendung. Die Gebrauchsfertigkeit für die Ausbildung stellt die Basis für den (mit weiteren Risiken verbundenen) Prozess der Schulung dar. Dessen Ziel besteht im Erreichen der Gebrauchsfertigkeit für den bestimmungsgemäßen Zweck. Diese Gebrauchsfertigkeit hat das neve Gesetz im Auge, nicht die für die weitere Fertigung.

\section{Minderung und Förderung der Nutzungskapazität}

Von dem im Gesetz für die Verbrauchsgüter gemeinten "Gebrauch" unterscheiden sich die Haltungs- und Trainingsmaßnahmen beim Pferd in einer weiteren Hinsicht: Der die Verbrauchsgüter betreffende "Gebrauch" stellt, wie zuvor skizziert, eine Reduktion des Verbrauchspotentials und damit auch des Wertes des Gutes dar. Demgegenüber sind die (unter anderem als Modi des "Gebrauchs" anzusprechenden) üblichen und außergewöhnlichen Ereignisse sowie die menschlichen Maßnahmen der Haltung, der Pflege und der Ausbildung in ihren Folgen ambivalent: Die Gebrauchsfertigkeit kann durch übliche und besondere gesundheitliche Prozesse, durch Unfälle, durch trainingsspezifische Krankheiten und Schäden sowie durch weitere Entwicklungen unerreichbar werden. Das Pferd kann sich - gegen alle Erwartungen und Pläne - aufgrund seiner mangelnden Leistungsfähigkeit und seiner mangelnden Leistungsbereitschaft als ungeeignet für den projektierten "Gebrauch" erweisen. In der größeren Zahl der Fälle fördern die Haltungs- und Trainingsmaßnahmen freilich die Gebrauchsfertigkeit für den eigentlichen Zweck, das heißt auch, sie steigern den Wert des Pferdes. Häufig tun sie dies aber nicht ohne Nebenfolgen, was wiederum bedeutet, dass die Förderung der Gebrauchsfertigkeit für den speziellen projektierten Zweck nicht selten mit gesundheitlichen Einbußen des Pferdes verbunden ist. Das besagt mit anderen Worten, dass die Förderung der speziellen Gebrauchsfertigkeit mit Einbußen des Gebrauchspotentials einhergeht. Zugleich können die fortschreitenden Ausbildungsmaßnahmen die Eignung des Pferdes für den projektierten Zweck in einem unerwarteten Maße deutlich werden lassen, also auch insofern das Gebrauchspotential und den Wert des Pferdes fördern. Häufig steht freilich der Förderung in manchen Hinsichten die Minderung in anderen gegenüber. Selbst beim zuvor angesprochenen 14-jährigen Jagdpferd sind nicht nur die üblichen Verschleiß- beziehungsweise "Verbrauchs"erscheinungen zu konstatieren; meist ist ein solches Pferd auch besonders "erfahren" und geeignet geworden, einen weniger qualifizierten Reiter heil über die Hecken, die Gräben und die Bäche zum Halali zu tragen. In manchen Fällen geht mit der qualitativen Verbesserung des Nutzungspotentials demnach dessen quantitative Verminderung einher.

Die mehr oder minder intensiven Einflüsse, denen bereits die Ei- und die Samenzellen, weiter die intrauterine Frucht, das Fohlen bei und nach der Geburt, das heranwachsende Pferd und das in der Ausbildung ausgesetzt sind, wirken sich, wie gesagt, im Potential des Pferdes für die eine oder die andere Nutzung mehr oder minder weitgehend aus. Sie wirken sich freilich ambivalent aus, fördern nämlich bald dieses Potential 
und reduzieren es bald. Häufig geht, wie ebenfalls schon gesagt, die Förderung in manchen Hinsichten mit der Minderung in anderen einher. Insofern sind diese Einflüsse nicht ausschließlich dem im Gesetz gemeinten Modus des "Gebrauchens", nämlich der Reduktion der Nutzungskapazität, zu subsumieren. Da diese Einflüsse und Einwirkungen die Nutzungskapazität aber nicht nur fördern beziehungsweise erschließen, sondern in der Regel zugleich mindern und insofern einen potentiellen "Neu"-Zustand aufheben, kann man wohl nicht umhin, die partielle Minderung beziehungsweise die Minderung in bestimmten Hinsichten als Folge eines "Gebrauchs" beziehungsweise einer Maßnahme zu verstehen, die in ihren Konsequenzen dem "Gebrauch" gleichkommt. Einen solchen Sachverhalt und eine solche Deutung hat man gewiss für die Maßnahmen anzunehmen, die für die Überführung des adulten Pferdes von seiner potentiellen Nutzbarkeit zur Gebrauchsfertigkeit erforderlich sind, nämlich für die Ausbildung zum Einsatz auf der Rennbahn, als Reitund/oder als Zugpferd.

Die Ei- und die Samenzellen sowie deren Verbindung existieren, wie gesagt, ebenfalls nicht in dem gebrauchsfertigen Neu-Zustand, den das Gesetz im Auge hat. Die Verwendung dieser "Sachen" für bestimmte genetische Maßnahmen stellt zudem eigentlich nicht den "Gebrauch" dar, den das Gesetz im Zusammenhang mit den "Verbrauchsgütern" anspricht. "Brauchbar" sind die Ei- und die Samenzellen sowie deren Verbindung nicht wie die üblichen "Verbrauchsgüter", nicht weitgehend unmittelbar, sondern nur unter recht komplexen Voraussetzungen, nicht regelmäßig und zuverlässig, sondern nur in einzelnen erfolgreichen Fällen, das heißt meist erst nach diversen Versuchen und Misserfolgen. Zudem werden die "brauchbaren" Zellen sowie deren Verbindung, wie ebenfalls schon gesagt, nicht in der im Gesetz unterstellten Weise "verbraucht", sondern gerade die "brauchbaren" und "gebrauchten" entfalten sich, bilden komplexere Organismen und überleben so - im Gegensatz zu den aufgebrauchten Verbrauchsgütern, die weitgehend wertlos werden, nämlich nur noch mehr oder minder recyclebares Material darstellen. Man weiß ferner von beträchtlichen Qualitäts- und Funktionsunterschieden der Zellen, auch von einem Qualitätsverlust, der mit dem Altern der Zellen verbunden ist. Für die Zellen existiert damit ebenfalls kein gebrauchsfertiger NeuZustand, insbesondere kein gebrauchsfertiger Neu-Zustand, der einem bestimmten, von einem eindeutigen Verwendungszweck definierten Standard entspricht.

Abschließend sei zu diesem Komplex angemerkt: Um Ungewissheiten bei der Zuordnung der Pferde zu den "neu hergestellten" oder den "gebrauchten" Verbrauchsgütern zu entgehen, könnte der als Unternehmer handelnde Verkäufer versuchen, das veräußerte Pferd beziehungsweise Fohlen im Kaufvertrag als "gebraucht" zu qualifizieren. Eine solche allgemeine Beschaffenheitsbeschreibung wird wahrscheinlich aber nicht rechtskräftig werden; man wird in ihr vielmehr die Absicht sehen, das gesetzliche Verbot der Verkürzung der Verjährungsfrist $(\S 475,2)$ zu umgehen. Erwähnt der (gewerbliche) Verkäufer demgegenüber im Vertrag einen (ohnehin offensichtlichen, vom Käufer bereits erkannten und meist als gebrauchsbedingt verstandenen) Mangel, zum Beispiel ein Überbein oder eine Narbe, dann ist dieser Mangel wohl als eine Folge "Gebrauchs" zu deuten, die Zuordnung des Fohlens zu den "gebrauchten" Verbrauchsgütern damit - unab- hängig von der hier erörterten allgemeinen Problematik - eindeutig und rechtsverbindlich.

\section{Resümee}

Die vorliegende Erörterung läuft darauf hinaus, Pferde generell, das heißt einschließlich der Fohlen, der intrauterinen Frucht, der Samen- sowie der Eizellen, den "gebrauchten Verbrauchsgütern" zuzuordnen. Diese Kategorisierung resultiert aus der gesetzlichen Vorgabe "gebrauchter" Verbrauchsgüter, der Alternative der "neu hergestellten Sachen" sowie dem Verständnis der Verbrauchsgüter als "gebrauchbarer" gleich "gebrauchsfertiger" Sachen. Sie resultiert weiter aus der Tatsache, dass bei Pferden - selbst bei den ausschließlich als Kumpanen oder als Rasenmähern eingesetzten und den zum Schlachten gezüchteten - ein gebrauchsfertiger Neu-Zustand nicht existiert.

Das Nutzungspotential der Pferde ist gewiss nach dem Erreichen ihrer Gebrauchsfertigkeit, das heißt nach ihrer Ausbildung für einen bestimmten Verwendungszweck, partiell oder sektoral geschmälert; insofern haben gebrauchsfertige Pferde gewiss den potentiellen "Neu"-Zustand verloren, das heißt sind sie "gebraucht".

Selbst wenn man den Zustand des Pferdes vor der Ausbildung (zunächst einmal) nicht eindeutig als "neu" oder als "gebraucht" versteht, muss man diesen Status als einen der (noch) nicht erreichten Gebrauchsfertigkeit bestimmen und damit eindeutig abheben von dem der neuen gebrauchsfertigen Güter, den der Gesetzgeber im Sinn hat. Da das Gesetz nur die Alternative "gebrauchter" und "never" gebrauchsfertiger Güter kennt, Pferde aber keine gebrauchsfertigen neuen Güter darstellen, könnten sie entweder neu, aber nicht gebrauchsfertig sein oder gebraucht und (weiterhin) gebrauchsfähig oder gebraucht und nicht (mehr) gebrauchsfähig, nämlich im Sinne des Gesetzes "verbraucht". Pferde letzterer Kategorie bleiben in den vorliegenden Überlegungen unberücksichtigt. Da die Pferde, die für die Klassifizierung als "neu" eventuell in Frage kommen, nicht gebrauchsfertig sind, ist deren die Zuordnung zu den neven und gebrauchsfertigen Gütern - logisch und nach dem Sinn des Gesetzes - auszuschließen. Ihre Klassifizierung als "gebraucht" wird aufgrund der gesetzlichen Alternative "gebraucht - neu" somit unausweichlich.

Die Klassifizierung "gebraucht" ist - im Rahmen der gesetzlichen Vorgaben und Absichten - selbst für junge Pferde sachlich gerechtfertigt, sofern man den juristischen Terminus des "Gebraucht"-Seins nicht (nur) als Synonym für das "Alt"-Sein oder den erfolgten bestimmungsgemäßen Gebrauch versteht, sondern mit ihm die Beeinträchtigung der Kapazität der bestimmungsgemäßen beziehungsweise der üblichen Nutzung anspricht. Die Klassifizierung ist im Rahmen der gesetzlichen Vorgaben und Absichten sachlich gerechtfertigt, weil die Einflüsse und die Einwirkungen auf die noch nicht befruchteten Zellen, auf den Embryo sowie auf das Fohlen und das heranwachsende Pferd dessen Nutzungspotential nicht nur fördern, sondern partiell auch schmälern und somit einen potentiellen "Neu"-Zustand aufheben, weil somit eine die Gebrauchsfertigkeit nicht berücksichtigende - Einstufung als "neu" selbst bei Zellen und Jungtieren problematisch blie- 
be, nämlich nicht grundsätzlich, sondern nur in Relation zu (intensiv genutzten) älteren Individuen vorgenommen würde. Dem Sinn des Gesetzes ist nicht dadurch zu entsprechen, dass man die für Lebewesen im allgemeinen und für Pferde im besonderen - unabhängig vom Problem der Grenzziehung - zutreffenden Begriffe "jung" und "alt" durch die im Gesetz genannten, zur Kennzeichnung des Zustandes von Lebewesen aber ungeeigneten, nämlich "nicht gebraucht" beziehungsweise "neu" einerseits und "gebraucht" andererseits, ersetzt. Die Subsumtion der Lebewesen im allgemeinen und die der Pferde im besonderen unter die (leblosen) Verbrauchsgüter und die sachlogisch inakzeptable Übertragung von Begriffen zur Kennzeichnung des Zustandes von (leblosen) Verbrauchsgütern auf Lebewesen zwingen dazu, über den Wortlaut des Gesetzes hinauszugehen und nach dessen Sinn zu fragen. Und dieser führt zu dem Schluss: Pferde sind in keinem Fall "neu". Sie sind zudem vor ihrer Ausbildung unter dem Sattel oder vor dem Wagen für ihren bestimmungsgemäßen Zweck (noch) nicht gebrauchsfertig.

Adulte Pferde, Fohlen, Ei- und Samenzellen sowie deren Verbindung generell nicht als "neu hergestellte" Sachen zu verstehen, sondern sie den "gebrauchten" Gütern zuzuordnen, dient nicht dem Zweck, die (für Verbrauchsgüter sinnvolle) Unterscheidung zwischen Nevem und Gebrauchtem zu umgehen, beziehungsweise es dient diesem Zweck nur insofern, als diese Unterscheidung für (die in der Regel differenziert genutzten) Pferde sachlich nicht zu rechtfertigen ist. Derart mit adulten Pferden, mit Fohlen, Ei- und Samenzellen sowie deren Verbindung zu verfahren, dient dem Zweck, die sachlich illegitime Übertragung der für leblose Verbrauchsgüter zutreffenden Begriffe und Verfahren auf differenziert genutzte Lebewesen so zu interpretieren, dass in der Rechtspraxis dem Sinn der EU-Richtlinie entsprochen werden kann.

Würde man Fohlen (bis zu einem bestimmten Alter) den neu hergestellten Verbrauchsgütern zuordnen und dadurch die Frist für die Haftung - im Vergleich zu der bei den ("gebrauchten") adulten Pferden - verlängern, so ergäbe sich die Frage nach dem Sinn einer solchen Maßnahme. Das heißt: Warum soll ein (professioneller) Verkäufer für die Beschaffenheit eines Fohlens länger haften als für die eines ausgewachsenen Pferdes? Versucht man einen solchen Unterschied mit den beim Fohlen noch nicht hinreichend erkennbaren "gebrauchsrelevanten" Eigenschaften zu begründen, dann müsste man die Haftung eigentlich bis zum bestimmungsgemäßen Gebrauch des adulten Pferdes ausdehnen. Eine solche, für den Verkäufer unbillige Haftung würde die sachlich ungerechtfertigte Prognose von den Eigenschaften des Fohlens auf die "Brauchbarkeit" des späteren Reit- oder Fahrpferdes voraussetzen und insofern unaufrichtiges Geschäftsgebaren einfordern und legitimieren. Das Fohlen lässt nämlich nur Vermutungen, aber keine sicheren Aussagen und keine haftungsrelevanten Zusagen über die "Gebrauchseigenschaften" des adulten Pferdes zu. Soll überhaupt für spezielle gebrauchsrelevante Eigenschaften eines Fohlens gehaftet werden oder nur generell für seine Gesundheit? Trifft letzteres zu, dann ergibt sich die Frage, ob die Gesundheit eines Fohlens zu ihrer Offenbarung eines längeren Zeitraums als die eines adulten Pferdes bedarf. Gegen das Konzept, den Haftungszeitraum für ein junges Pferd zu verlängern, wäre die These zu stellen: Die Gebrauchseigenschaften eines adulten Pferdes lassen sich leichter erkennen als die eines noch nicht gebrauchsfertigen, noch nicht im Gebrauch testbaren Fohlens. Daher kann ein Verkäufer die Gebrauchseigenschaften eines gesunden adulten Pferdes seriöser zusagen als die eines Fohlens. Der Verbraucher kann diese Eigenschaften seriöser erwarten, der Verkäufer kann seriöser für sie haften. Speziell kann der Verkäufer die (zum Zeitpunkt des Verkaufs gegebenen) Gebrauchseigenschaften des zuvor angesprochenen 14-iährigen Jagdpferdes seriöser und auch haftungsrelevant zusagen als die des Fohlens, dessen Pedigree, dessen Exterieur und dessen Bewegungsablauf - vor allem in der Anpreisung des Verkäufers - "ein Pferd für höchste Ansprüche" "erwarten" lässt.

Stellt man die verschiedenen Argumente für oder gegen eine unterschiedliche Haftungsdaver beim Kauf von Samenzellen, von Fohlen oder von adulten Pferden redlich einander gegenüber, so lässt sich meines Erachtens eine längere Frist bei den jungen Lebewesen nicht rechtfertigen. Man müsste wahrscheinlich eher für eine kürzere als für eine längere Haftung bei den als "neu" eingestuften Jungtieren plädieren. Generell ergibt sich hier jedoch kein Sinn, der dem der unterschiedlichen Haftungsdauer bei neu hergestellten Verbrauchsgütern einerseits und gebrauchten andererseits entspricht. Hat man bei den Pferden - wie bei den Verbrauchsgütern - das unterschiedliche Nutzungsrisiko beziehungsweise das unterschiedliche Nutzungspotential des Neven und des Gebrauchten im Auge, dann scheitert die Übertragung von den leblosen Verbrauchsgütern auf die Pferde schon deshalb, weil die Brauchbarkeit des Fohlens für den bestimmungsgemäßen Zweck sich, wie gesagt, seriös nicht prognostizieren und dem Verbraucher zusagen lässt, weil zudem ein bemerkenswerter Anteil der Fohlen die Gebrauchsfähigkeit für den bestimmungsgemäßen Zweck nicht erreicht und das (restierende) Gebrauchspotential mancher 14-iähriger Jagdpferde beträchtlich über dem liegt, das manche Fohlen in ihrer späteren Karriere unter dem Sattel zur Verfügung stellen.

Ordnet man die adulten Pferde ebenso wie Fohlen, einzelne Zellen und Zellverbindungen nicht den "neu hergestellten", sondern den "gebrauchten" Sachen zu, dann beschränkt man die Haftungsdaver des als Unternehmer tätigen Verkäufers gegenüber dem Verbraucher auf ein Jahr. Im Vergleich zur Zwei-Wochen-Frist in der Kaiserlichen Verordnung wird der Schutz des Verbrauchers also erheblich verstärkt. Zudem reicht die Frist von 12 Monaten in der Regel aus, um gebrauchsrelevante Mängel eines adulten Pferdes zu offenbaren. Selbst der Zeitraum von einem Jahr ist für die Daver der Haftung beträchtlich. Er ist nicht unproblematisch, weil in dieser Zeit die Auswirkungen der verschiedenen Modi des "Gebrauchs" erheblich sein können und damit die Schwierigkeiten wachsen, den Zustand des Pferdes ohne diese Auswirkungen, nämlich den Zustand des Pferdes bei dessen Übernahme durch den Verbraucher, zu beweisen. Diese Schwierigkeiten würden sich mit der Verdoppelung des Haftungszeitraums auf zwei Jahre quasi potenzieren, und zwar mit der Folge komplexer juristischer Auseinandersetzungen, in denen die Überzeugungskraft der Gutachter schwerer wiegen könnte als die juristische Entscheidung für Gesetzeskonformität und Gerechtigkeit.

Mit Hilfe grundsätzlicher Überlegungen und Kommentare, und nicht nur über Sachverständigengutachten und Einzelfallentscheidungen im Rahmen der Rechtspraxis (Fellmer et al. 2002,33), wird in Zukunft das Gesetz zum Verbrauchsgüter- 
kauf für den Handel mit Pferden praktikabel zu machen sein, und zwar ein Gesetz, das die EU-Richtlinie zum Kauf von "Verbrauchsgütern" generell auf "Sachen" ausdehnt, das Tiere implizit mit den für leblose Verbrauchsgüter zutreffenden Kategorien beschreibt und die den Lebewesen eigenen biologischen Gesetzlichkeiten ignoriert - letztlich also ein Gesetz, das in einer - für die nahe Zukunft allerdings nicht anstehenden - Novellierung weitergehend als in seiner derzeitigen Fassung mit der Wirklichkeit in Einklang zu bringen wäre.

\section{Danksagung}

Für wichtige Hinweise auf die juristische Literatur und die juristische Sicht danke ich Dr. Dietrich Plewa (Germersheim) und Dr. Joachim Wann (Warendorf).

\section{Literatur}

Brückner S und Böhme A (2002): Neues Kaufrecht - wann ist ein Tier "gebraucht"? in: Monatsschrift für Deutsches Recht, Vorabdruck

Fellmer E, Brückner S und Rahn A (2002): Das völlig neve Pferdekaufrecht ab 1. Januar 2002. Warendorf

Freudenreich W G (1990): Junge Hunde als "neu hergestellte Sachen". In: Neue Juristische Wochenschrift Heft 14/1990
Henssler M, Hrsg. (2001): Praxis der Schuldrechtsreform. Recklinghausen

Hoeren T, Hrsg. (2002): Systematischer Kommentar zum Kaufrecht. Recklinghausen

N N (1986): Ausschlußfrist für Mängelanzeige bei nicht offensichtlichen Mängeln. In: Neue Juristische Wochenschrift-RR, Heft $1 / 1986$

N N (2001): Münchener Kommentar zum Bürgerlichen Gesetzbuch, Bd. 1. 4. Aufl. München

Palandt, N N (1998): Bürgerliches Gesetzbuch, Bd.7. 5.Aufl. München

Plewa D (2002): Die Kaufuntersuchung des Pferdes aus rechtlicher Sicht. In: Pferdeheilkunde 18,

Weber H J, Dospil J und Hanhörster H (2002): Mandatspraxis Neues Schuldrecht. München

Wolf M, Horn N und Lindacher W (1999): AGB-Gesetz. 4. Aufl. München

Prof. Dr. phil. Heinz Meyer

Am Wisselsbach 22

52146 Würselen 